KARL JANSEN-WINKELN

\title{
Drei Statuen der 22./23. Dynastie
}

\author{
Hierzu Tafel VI-XVI
}

Im Folgenden sollen drei unveröffentlichte Statuen behandelt werden ${ }^{1}$, die im Museum Kairo bisher wohl wenig beachtet worden sind, da alle drei erheblich beschädigt sind: der ersten (JE 37884) fehlt der Kopf, die zweite (TN 20/2/ 15/1) hat durch Feuchtigkeit stark gelitten, so dass die meisten ihrer Inschriften verloren sind, und bei der dritten (TN 20/2/25/2) fehlt der ganze untere Teil. Dennoch lassen sich alle drei zeitlich sicher einordnen, bei den beiden letzteren ist sogar der Besitzer von anderen Denkmälern her bekannt.

\section{Stehender Theophor Kairo JE 37884 (= JE 38633)}

Die Statue stammt aus der Cachette von Karnak (Fundnummer K.527), sie ist aus Kalkstein und noch $32 \mathrm{~cm}$ hoch (Sockel 18,3 cm tief und ca. $8 \mathrm{~cm}$ breit). Ein stehender Mann, in einen langen Faltenschurz mit Pantherfell darüber gekleidet und mit Sandalen, deren Querriemen deutlich ausgeprägt sind, hält mit beiden Händen eine Figur des Gottes Amun vor sich, die auf einem kleinen Schrein mit Hohlkehle steht. Der Gott ist gleichfalls mit einem Faltenschurz bekleidet, der aber nur bis zu den Knien reicht. Er trägt die Doppelfederkrone und hält mit der linken Hand ein Szepter vor sich. Der Kopf des Priesters fehlt, und an mehreren Stellen der Statue, vor allem der rechten Seite und am Sockel, sind kleinere Teile weggebrochen. Auf dem Rückpfeiler ist eine fast vollständige Inschrift von zwei Kolumnen (die letzten drei

\footnotetext{
${ }^{1}$ Für die Erlaubnis zur Veröffentlichung danke ich der Generaldirektorin des Ägyptischen Museums Kairo, Frau Dr. Wafaa el-Sadeek.
}

Gruppen der zweiten Spalte fehlen), und an seiner linken Seite, etwa in der Mitte, befindet sich eine einzelne kürzere Kolumne.

JE 37884 ist in $\mathrm{PM} \Pi^{2}, 159$ (mit falschem Namen) aufgelistet, wird aber $\mathrm{m}$. W. sonst in der Literatur nicht erwähnt.

Die Figur selbst gibt auf den ersten Blick noch keinen Anhaltspunkt für eine genauere Datierung. Aufschlussreicher ist der Stammbaum, der sich, den Stifter eingeschlossen, über acht Generationen erstreckt (vgl. Fig. 1, links). Besitzer ist ein $h m-n t \underline{n} n$ Jmn-R $R^{\top} n j s w t n t r w$ und hrj-š̌t n pr Jmn namens P3-ḩ3rw. Seine nächsten fünf Vorfahren sind alle $h m-n t \underline{t r} n \mathrm{Jmn}$ und $h r j$ $s \check{s} t 3$, der Urururgroßvater $H n z w-m s j w$ genauer hrj-sšt jumj pr $n$ Jmn. Dessen Vater, der älteste genannte Vorfahr, war allerdings nicht $h m-n t r r$ $J m n$, sondern jtj-ntrr mrjj(-nțr) und $h r j-s \check{t} t 3 n$ mnw nb $n$ Jmn [. . .]. Stifter der Statue ist ein $\mathrm{Hr}$ mit

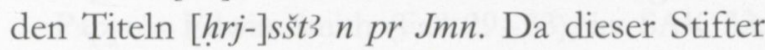
der Sohn eines hmm-ntrr $n$ Jmn hrj-š̌t $n$ n pr Jmn namens $P 3-h 3 r w$ ist, handelt es sich gewiss um den Sohn des Besitzers, obwohl das nicht ausdrücklich gesagt wird.

Vier Generationen vor dem Besitzer und fünf vor dem Stifter trifft man auf einen $h m-n t r r ~ n J m n$ und $h r j-s s ̌ t 3$ namens $` h 3-n f r-J m n$ mit dem Beinamen P3-h3rw. Ein Mann mit eben diesem Namen und Beinamen sowie - neben vielen anderen - diesen Titeln ist im Massengrab der „Bab el-Gasus“ bestattet worden (Sargensemble A.115) ${ }^{2}$, und zwar etwa in der Zeit des Hohen-

\footnotetext{
${ }^{2}$ Name, Beiname und die meisten Titel finden sich auf seinem Sargensemble Kairo JE 29670 (s. A. Niwiński, $21^{\text {st }}$ Dynasty Coffins from Thebes. Chronological and Typological Studies, Theben V, 1988, 125, Nr. 113), seinem Leichentuch (ASAE 8, 1907, 31-2) und dem Papyrus Kairo JE 95878 (s. Piankoff, Litany
} 
JE 37884:

Bab el-Gasus A.114:

A. 115

$$
\text { (pTN 23/4/40/3) }
$$

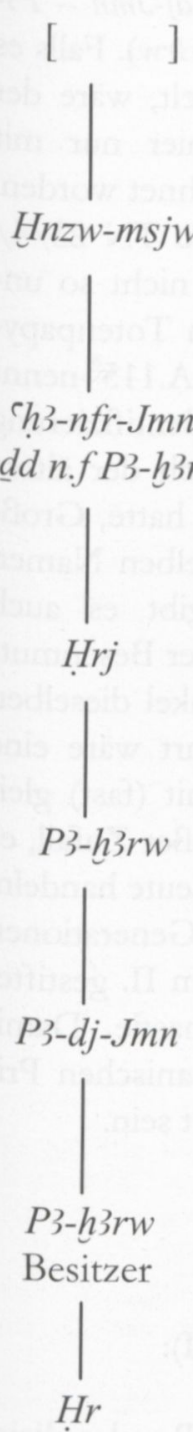

Stifter

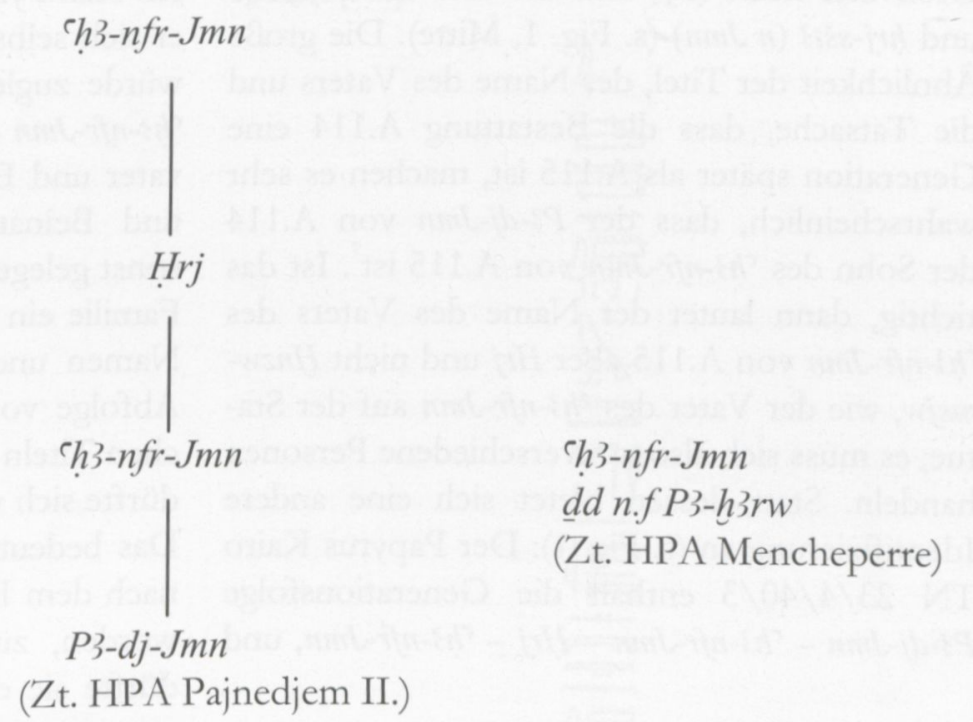

Fig. 1. Familie des Pacharu priesters Mencheperre, dessen Name auf den Mumienriemen erscheint ${ }^{3}$. Wäre dies derselbe Mann wie der Vorfahre des Statuenbesitzers, wie

of Re, 66-71; 133-7). Sein anderer Totenpapyrus Kairo JE 95705 scheint weniger informativ zu sein, s. A. Niwiński, Studies on the Illustrated Theban Funerary Papyri of the 11th and 10th Centuries B.C., OBO 86, 1989, 299 (129).

ASAE 8, 32. Einige Begräbnisse enthalten Objekte (wie Mumienriemen oder Leichentücher) mit den Namen oder Daten mehrerer Herrscher (z. B. ASAE 8, 31 [113], 33 [124]. Das Begräbnis muss also nicht zwin- man zunächst annehmen möchte, sollte die Statue etwa fünf Generationen nach Mencheperre hergestellt worden sein. Man käme so etwa auf die Zeit von Osorkon I. bis (spätestens) die ersten Jahre Osorkons II. Diese Gleichsetzung wird aber durch eine zweite Bestattung in demselben Massengrab unwahrscheinlich. Das Be-

gend in die Regierung des Mencheperre fallen, es dürfte aber zumindest zeitlich nicht allzu weit von ihr entfernt sein. 
gräbnis A.114 ${ }^{4}$ gehört einem $P 3-d j-J m n^{5}$, der ganz ähnliche Titel trug wie der ' $h 3-n f r-J m n$ von A.115 und etwa in der Zeit des Hohenpriesters Pajnedjem II. begraben wurde, des Sohnes und Nachfolgers des Mencheperre. Unter den Grabbeigaben enthält nur der "mythologische" Papyrus Kairo 23/4/40/3 eine Filiationsangabe; er führt sogar - recht ungewöhnlich für einen Totenpapyrus dieser Zeit - Vater, Großvater und Urgroßvater des Verstorbenen an. Der Vater und der Urgroßvater heißen ' $h 3-n f r-J m n$, der Großvater heißt $H r j$, und alle drei waren $j t j-n t r$ und hrj-sšt3 (n Jmn) (s. Fig. 1, Mitte). Die große Ähnlichkeit der Titel, der Name des Vaters und die Tatsache, dass die Bestattung A.114 eine Generation später als A.115 ist, machen es sehr wahrscheinlich, dass der P3-dj-Jmn von A.114 der Sohn des ‘ h3-nfr-Jmn von A.115 ist ${ }^{7}$. Ist das richtig, dann lautet der Name des Vaters des Ch3-nfr-Jmn von A.115 aber Hrj und nicht Hnzwmsjw, wie der Vater des `h3-nfr-Jmn auf der Statue; es muss sich also um verschiedene Personen handeln. Statt dessen bietet sich eine andere Identifizierung an (s. Fig. 1): Der Papyrus Kairo TN 23/4/40/3 enthält die Generationsfolge P3-dj-Jmn - `h3-nfr-Jmn - Hrj- ${ }^{\top} h 3-n f r-J m n$, und

\footnotetext{
${ }^{4}$ Also vermutlich neben oder nahe bei A.115. Es lässt sich mehrfach beobachten, dass die Nummern der Begräbnisse verwandter Personen nicht weit voneinander entfernt sind, und damit vermutlich auch die Särge selbst. So haben etwa zahlreiche Nachkommen des Hohenpriesters Mencheperre besonders hohe Nummern und waren daher wohl ganz im Innern dieser Cachette bestattet.

Sargensemble Kairo JE 29666, s. Niwiński, $21^{\text {st }}$ Dynasty Coffins from Thebes, 124, Nr. 109; funeräre Papyri Kairo TN 23/4/40/2 (s. Piank off, Myth. Pap., Nr. 10) und Kairo JE 95879 (s. Niwiński, Studies on the Illustrated Theban Funerary Papyri, 268 [44]); Leichentuch, s. ASAE 8, 31.

Der Name des Pajnedjem steht auf den Mumienriemen, s. ASAE 8, 31.

Da der Hohepriester Mencheperre fast 50 Jahre regiert hat und sein Sohn Pajnedjem II. deutlich länger als 20 Jahre amtierte, wäre es theoretisch nicht ausgeschlossen, dass es sich bei dem ${ }^{h} 3-n f r-J m n$ von A.115 nicht um den Vater, sondern um den Urgroßvater des P3-dj-Jmn handelte. Das ist aber sehr unwahrscheinlich, umso mehr, als die Bestattungen dieses Massengrabes wohl nur die letzten drei Generationen der 21. Dynastie umfassen, und damit keine aus den frühen Jahren des Mencheperre.
}

aus der Bestattung A.115 wissen wir, dass der jüngere ' h3-nfr-Jmn den Beinamen P3-h3rww führte. Auf der Statue JE 37884 heißen die vier auf den Besitzer folgenden Ahnen P3-dj-Jmn - P3$h 3 r w-H r j-c h 3-n f r-J m n(d d n . f P 3-h 3 r w)$. Falls es sich um dieselben Personen handelt, wäre der Großvater des Statuenbesitzers hier nur mit seinem „Beinamen“ $P 3$ - h3rw bezeichnet worden, während ihn sein Sohn (auf pKairo TN 23/4/ 40/30) Ch3-nfr-Jmn nennt. Das ist nicht so ungewöhnlich, denn auch auf seinem Totenpapyrus Kairo JE 95705 aus Begräbnis A. $115^{8}$ nennt er sich selbst nur P3-h3rw. Diese Identifizierung würde zugleich bedeuten, dass auch der ältere Ch3-nfr-Jmn den Beinamen P3-h3rw hatte, Großvater und Enkel führten also dieselben Namen und Beinamen. Vergleichbares gibt es auch sonst gelegentlich, z. B. tragen in der BesenmutFamilie ein Priester und sein Urenkel dieselben Namen und Beinamen'. Umgekehrt wäre eine Abfolge von vier Generationen mit (fast) gleichen Titeln und Namen ein zu großer Zufall, es dürfte sich wirklich um dieselben Leute handeln. Das bedeutet, die Statue ist zwei Generationen nach dem Hohenpriester Pajnedjem II. gestiftet worden, zu Beginn der 22. Dynastie. Damit dürfte sie eine der frühesten thebanischen Privatstatuen der Dritten Zwischenzeit sein.

\section{Übersetzung}

\section{Rückseite (Fig. 2, Tf. VI):}

1 Ein Königsopfer des Amun-Re, der diejenigen bedrängt, die diesen Tempel nicht betreten, damit sie alles geben ${ }^{10}$, was auf ihren (der

\footnotetext{
${ }^{8}$ S. Niwiński, Studies on the Illustrated Theban Funerary Papyri, 299 (129).

Auf dem Sarg Kairo CG 41046, s. dazu K. A. Kitchen, The Third Intermediate Period in Egypt (1100650 BC), 3. Auflage, Warminster 1996, \ 190.

${ }^{10}$ Eine Opferformel mit ganz ungewöhnlichem Beginn: das Epitheton des Gottes ist offenbar eine indirekte Drohung gegen diejenigen, die der Statue nich opfern. Die Reihenfolge der Zeichen ist vertauscht worden: $₹ q$ muss vor $p r p n$ zu lesen sein, und die Gruppe unter $₹ q$ kann nur eine Umstellung aus dj.sn sein.
} 


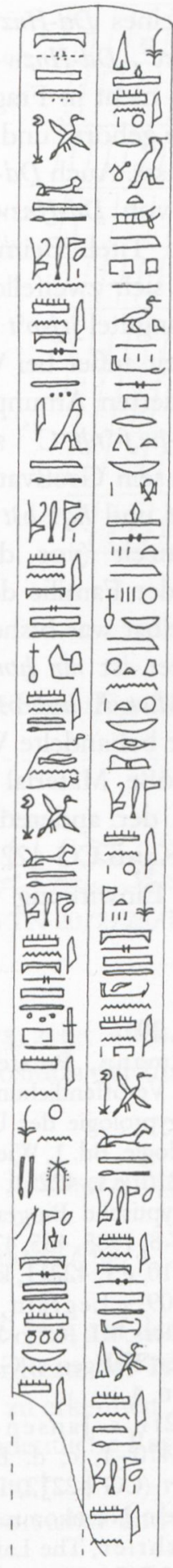

Fig. 2. Kairo JE 37884, Rückseite

Götter) Altar kommt an allen guten Dingen tagtäglich 〈für〉 den Osiris, den Propheten des Amonrasonther, den Eingeweihten des Hauses des Amun P3-h3rw, gerechtfertigt, den Sohn des Propheten des Amun und Eingeweihten P3-dj-
Jmn, gerechtfertigt, des Sohnes des Propheten des Amun 2 und Eingeweihten P3- $h 3 r w$, gerechtfertigt, des Sohnes des Propheten des Amun und Eingeweihten $\mathrm{Hrj}$, gerechtfertigt, des Sohnes des Propheten des Amun und Eingeweihten Ch3-nfr-Jmn, gerechtfertigt, genannt P3-h3rw, gerechtfertigt, des Sohnes des Propheten des Amun und Eingeweihten, der im Hause des Amun ist, Hnzw-msjw, gerechtfertigt, des Sohnes des Gottesvaters und $m r j j(-n \underline{t} r)$, des Eingeweihten aller Denkmäler des [Hauses des?] Amun [...].

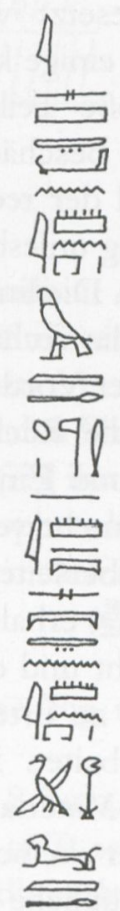

Fig. 3. Kairo JE 37884, linke Seite des Rückenpfeilers

Linke Seite des Rückenpfeilers (Fig. 3, Tf. VIII):

(Gemacht) durch den Eingeweihten ${ }^{11}$ des Hauses des Amun $\mathrm{Hr}$, gerechtfertigt, den Sohn des Propheten des Amun und Eingeweihten des Hauses des Amun P3-h3rw, gerechtfertigt.

\section{Würfelhocker Kairo TN 20/2/25/1 $=$ JE 37382}

Die Statue (aus der Cachette von Karnak, Fundnummer K.149) ist aus Kalkstein und

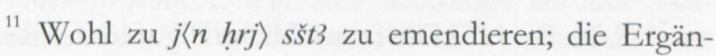
zung eines jrij (o. ä.) ,gemacht" ist nicht zwingend erforderlich. 
$46,4 \mathrm{~cm}$ hoch; der Sockel ist $23,3 \mathrm{~cm}$ tief, $23,2 \mathrm{~cm}$ lang und ca. $6,0 \mathrm{~cm}$ hoch.

Die Figur sitzt auf einem Sockel, der eine kleine Stufe im vorderen Bereich hat. Die Körperformen sind wenig ausgeprägt, die Füße waren nicht frei. Die linke Hand liegt flach aus dem Knie, die rechte hält eine gemusterte Pflanze. Das Gesicht zeigt die für Würfelhocker der Dritten Zwischenzeit typischen Züge ${ }^{12}$, die Strähnenperücke lässt die Ohren frei, der Rückenpfeiler ist ungewöhnlich breit. Die Statue war in mehrere Teile zerbrochen und ist modern wieder zusammengesetzt worden, ein großes Stück der Füße und einige kleinere Teile fehlen. Außerdem sind große Teile der Statue durch Feuchtigkeit schwer beschädigt, die Inschriften der Vorderseite und der rechten Seite sind dadurch fast vollständig unlesbar, ebenso die linke Hälfte der Rückseite. Die linke Seite ist mit neun Zeilen beschriftet, die rechte Seite war es vermutlich auch. Auf der Vorderseite sind nur einige Reste erhalten, die Rückseite enthält einige Zeilen, Kolumnen und Einzelzeichen in merkwürdiger und nicht mehr verständlicher Anordnung, und auf der Oberseite des Sockels links ist noch eine Gruppe (S) erhalten.

Nach dem Gesicht und den noch erhaltenen Teilen der Inschrift zu urteilen, war die Statue sehr sorgfältig gearbeitet. Das wird allerdings auch etwas mit dem Material zu tun haben, denn Kalkstein ist leichter zu bearbeiten als der für Würfelhocker sonst übliche Hartstein.

Die Statue ist unpubliziert und wird $\mathrm{m}$. W. nicht in der Literatur erwähnt.

Besitzer:

Dd-Hnzw-jw.f- ${ }^{-} n h \quad$ hm-ntr 4-nw n Jmn [m Jpt-swt](?) $j r j-p^{\top} t h 3 t j-{ }^{\top}$

htmtj bjtj smr wetj

$m r$ htmt

$h q 3$ b3t

(m) $r$ hwt-ntr $n t$ Jmn shd himw-ntr nbw

Vierte Propheten des Amun dieses Namens sind in der Dritten Zwischenzeit mehrfach belegt: Gesichert sind $\underline{D} d-H n z w-j w . f-{ }^{-} n h$ A, C und

${ }^{12}$ S. K. Bosse, Die menschliche Figur in der Rundplastik der ägyptischen Spätzeit von der XXII. bis zur XXX. Dynastie, ÄF 1, 1936, 26 (42).
$\mathrm{D}$, die Existenz eines $\underline{D} d-H n z w-j w . f-{ }^{-} n h \mathrm{~F}$ hat Bierbrier postuliert ${ }^{13} . D d-H n z w-j w . f-{ }^{-} n h$ D (und ebenso F) kommt nicht in Frage, weil er schon in die 25. Dynastie gehört ${ }^{14}$ und auch von seinen Titeln her nicht passt. Auch $\underline{D} d-H n z w-j w . f-{ }^{-} n h$ A, der Urgroßvater von $D d-H n z w-j w . f^{-} n h$ C, hat wesentlich andere Titel. Beim Besitzer dieser Statue handelt es sich zweifellos um $\underline{D} d$-Hnzw$j w . f-{ }^{\ulcorner} h$ C: Die Rangtitel jrj-p ${ }^{\top} t h 3 t j-{ }^{-}$etc. sind in der 3. Zwischenzeit außer bei Weziren ziemlich selten; von den vierten Amunpropheten tragen sie nur Dd-Hnzw-jw. $f^{-} n h C^{15}$ sowie sein Vater $H r-z 3-3 s t \mathrm{C}^{16}$ und sein Großvater Nht.f-Mwt $\mathrm{A}^{17}$. Die Titel $m r$ htmt und $h q 3$ b3t kommen in der Dritten Zwischenzeit (vor der 25. Dynastie) ausschließlich in der Familie des Dd-Hnzw-jw.f' $n h \mathrm{C}$ vor $^{18}$. Er selbst war bisher nur als $h q 3$ b3t belegt ${ }^{19}$, sein Vater ist $m r h t m t h q 3 b 3 t^{20}$, sein Großvater Nht.f-Mwt A $h q 3 b 3 t^{21}$ bzw. $m r h t m t$ $h q 3 b 3 t^{22}$. Der hier behandelte Würfelhocker ähnelt zudem in Größe, Material und Qualität der Ausführung sehr der anderen Statue des $\underline{D} d$ Hnzw-jw. $f^{-} n h$ C, Kairo CG 42211, und wie diese hat er unter der Einwirkung von Feuchtigkeit

${ }^{13} \mathrm{BiOr} 36,1979,307$.

Vgl. G. Vittmann, Priester und Beamte im Theben der Spätzeit. Veröffentlichungen der Institute für Afrikanistik und Ägyptologie der Universität Wien, 3. Beiträge zur Ägyptologie, Bd. 1, Wien 1978, 17-8; 96.

${ }^{15}$ Kairo CG 42210, c,1; 42211, e,1; k,1, s. K. Jansen-Winkeln, Ägyptische Biographien der 22. und 23. Dynastie, ÄUAT 8, 1985, 465; 471; 475.

Kairo CG 42210, e,1; 42211, k,2, s. ibid., 466; 475.

${ }^{17}$ Kairo CG 42209, s. Legrain, Statues et statuettes de rois et de particuliers, III, Kairo 1914, 24.

${ }^{18} \mathrm{Zu}$ den späteren Belegen s. G. Vittmann, Priester und Beamte, 11, n. 4.

${ }^{19}$ Kairo CG 42211, s. Jansen-Winkeln, Ägyptische Biographien, 470-1, c; d. Ebenso nur $h q 3$ b3t nennt sich T3j-n-hsrt (CG 42212, Legrain, Statues et statuettes, III, 33), ein Nachkomme des Dd-Hnzw-jw.f' $n h$ C, s. M. L. Bierbrier, The Late New Kingdom in Egypt (c. 1300 664 B.C.), Warminster 1975, 84.

Quibell-Spiegelberg, Ramesseum, pl. XXIII,5; Statuen Karnak T 35, rechte Seite oben, s. KarnakNord IV, Fig. 143; CG 42210, b,6, s. Jansen-Winkeln, Ägyptische Biographien, 463.

CG 42209, e; 42211, k,3, s. Legrain, Statues et statuettes, III, 24; 31 .

${ }^{22}$ Karnak T 35, Vorderseite, oben, s. Karnak-Nord IV, Fig. 140; CG 717, rechts, s. Mariette, Karnak, pl. 47 e. 


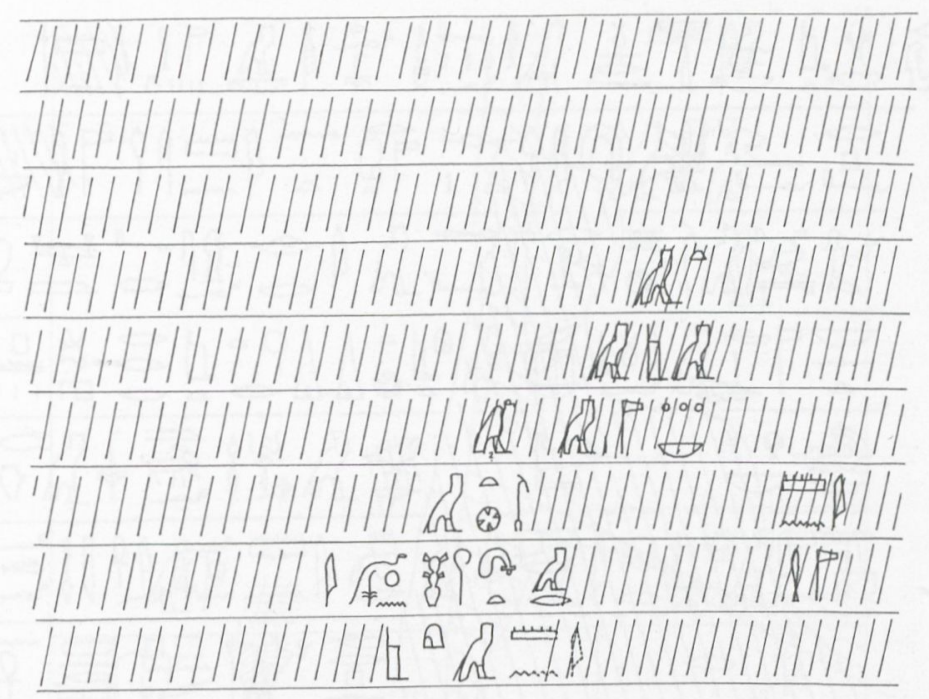

Fig. 4. Kairo TN 20/2/25/1, Vorderseite

Rechte Seite (Fig. 5, Tf. X):

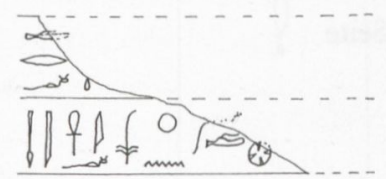

Fig. 5. Kairo TN 20/2/25/1, rechte Seite

gelitten. Überdies zeigt auch CG 42211 eine eigenwillige Disposition der Inschriften ${ }^{23}$.

\section{Übersetzung}

Vorderseite (Fig. 4, Tf. IX):

In vermutlich $9[+\mathrm{x}$, auf den nicht erhaltenen Füßen] Zeilen nur Reste der Titulatur erhalten:

Z. 1-3: nichts zu erkennen

Z. 4-6: nur undeutliche Reste

$7[\ldots]$ Theben in $[\ldots]$

8 [...] Schatzvorsteher und $h q 3$ b3t Dd-Hnzw$j\left[w . f-r_{n h} \ldots\right]$

9 [...A]mun in Karnak [. . . ]

Die Zeilen 1-7(?) sind bis auf undeutliche Spuren einiger Zeichen unlesbar. Bei langem

${ }^{23}$ S. dazu auch H. G. Fischer, The Orientation of Hieroglyphs, I, Reversals, New York 1977, 27-9. und gründlichem Studium des Originals ${ }^{24}$ unter günstigen Lichtverhältnissen wären vielleicht ein paar Zeichen mehr zu entziffern, jedenfalls keine zusammenhängenden Phrasen.

Z. 8(?): [... ohne(??)] ihn zu vertreiben,

Z. 9(?) [... 4. Prophet des Amun in Karn]ak Dd-Hnzw-jw.f- $n h$, gerechtfertigt

\section{Linke Seite (Fig. 6, Tf. XI):}

1 ,Osiris ist dein Schutz, Isis ist dein Schirm, der Pfeil des Horus trifft seinen Leib, (nämlich) desjenigen, der sich dir in den Weg stellen wird ${ }^{25}$, 4. Prophet des Amun [in Karnak], 2 Erbfürst und Graf, königlicher Siegler, einzigartiger Freund, Vorsteher ${ }^{26}$ des Tempels des Amun, Aufseher aller Propheten, 3 der in demütiger

${ }^{24}$ Zurzeit nicht möglich, da die Statue jeweils nur für sehr begrenzte Zeit aus der Vitrine genommen werden darf.

${ }^{25} \mathrm{Zu}$ d $3 j$,treffen“ s. Wb V, 515,2, zu ,sich jdm. in den Weg stellen“ ibid., 514,14. Die eigentliche Bedeutung in beiden Fällen ist „kreuzen“; es wird sich um ein Wortspiel handeln.

${ }^{26} \mathrm{Zu}$ dieser Schreibung von $m r$ „Vorsteher" s. Ch. Kuentz, BIFAO 34, 1934, 154-7. Der Titel selbst ist in dieser Zeit äußerst selten, ebenso der folgende. Dd-Hnzw-jw.f-enh C legt sich allerdings auf der Vorderseite seiner Statue Kairo CG 42211 ebenfalls eine Reihe von recht seltenen Titeln zu, s. Jansen-Winkeln, Ägyptische Biographien, 471. 


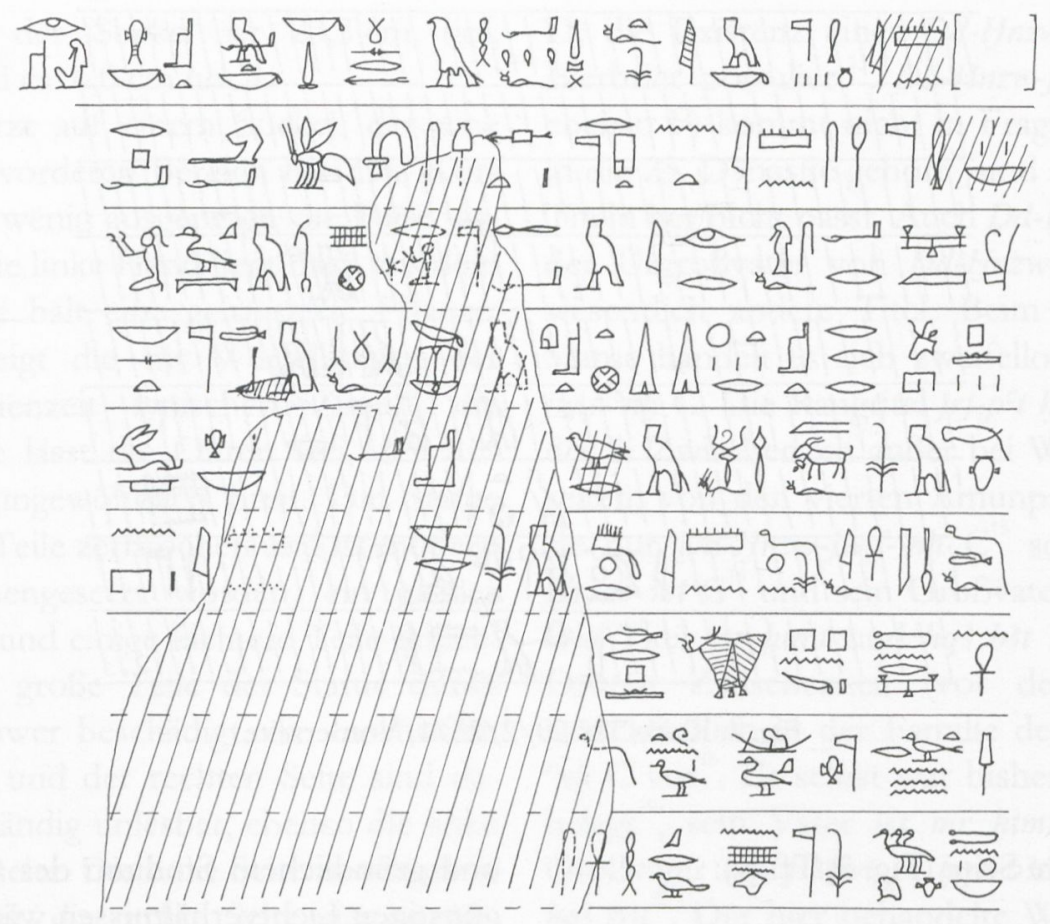

Fig. 6. Kairo TN 20/2/25/1, linke Seite

Haltung den ,Himmel ${ }^{6}$ betritt in Theben ${ }^{27}$, [um anzubeten(? $)^{28}$ ] den großen [Gott] in seiner Gestalt, der die Maat gelangen lässt zu 4 ihrem Herrn, der den Gott bekleidet mit seinem Gewand, der Festleiter [in] Karnak, der die Dinge an ihre (richtige) Stelle legt ${ }^{29}$, erfahren 〈in〉 den Gesetzen $^{30}, 5$ klug, ein Herr von [...] groß in seinem Amt, bedeutend in seinem Rang, der Vertraute des Königs ${ }^{31} \mathbf{6}$ im Haus des A[mun ... .] der(?) ihn liebt als Ehrwürdigen bei Amun, Dd-Hnzw-jw. - $^{-} n h$, gerechtfertigt, indem er sagt: 7 [,O ihr Eintretenden (o. ä.) ...] die ihr speist den Würdigen $^{32}$ dieses Hauses: Ich will euch auf

\footnotetext{
${ }^{27} h f{ }^{c} q$ wörtlich wohl, ,der in demütiger Haltung ist und betritt".

${ }^{28}$ Ein Zacken des Sterns $(d w 3)$ könnte noch erhalten sein, aber das ist nicht sicher.

${ }^{29}$ Zweifellos im Sinne von ,der alles richtig macht“.

${ }^{30}$ Eine Parallele aus dieser Zeit auf Kairo CG 42226, Rückseite, Z. 5, s. Jansen-Winkeln, Ägyptische Biographien, 510.

${ }^{31}$ Das $m$ gehört mit zu der (altertümlichen) Schreibung von $m h(-j b)$, s. Wb II, 116, und die Belegstellen zu Wb II, 118, 13; 15.

${ }^{32}$ Die Hieroglyphe stellt den Halsschmuck des Hohenpriester von Memphis dar, s. dazu Ch. Maystre,
}

den Weg des Lebens führen ${ }^{33} 8$ [...], wenn man spricht(?) zu (meinem) Sohn dort(?), der beim Gott eintritt, indem er rein ist, der leitet(?) $9[\ldots$ um] am Leben [zu erhalten $]^{34}$ den Hungrigen (meines[?]) Gaues, indem das Ansehen des Gottes und die Macht des Königs in(?) euren Herzen sind ${ }^{35}$ täglich."

Les grands prêtres de Ptah de Memphis, OBO 113, 1992, pl. I (Hinweis M. Eaton-Krauss), und ist $s^{c} h \mathrm{zu}$ lesen, s. Wb IV, 50, 5; A. Erman, ZÄS 33, 1895, 22-3. Hier kann es eigentlich nur eine $(\mathrm{m}$. W. sonst nicht bezeugte) bildliche Schreibung von $s h$ „der Würdige“" u. ä. sein, denn in seiner eigentlichen Bedeutung, als Halsschmuck, gehört dieses Objekt ja nicht zu ,diesem Haus", dem Amuntempel von Karnak. Allerdings ist in dieser Zeit merkwürdigerweise dieser Halsschmuck auch einmal in einer Grabkapelle in Theben belegt, und zwar in der des Großvaters des Besitzers dieser Statue (Nht.f-Mwt A, s. Quibell-Spiegelberg, Ramesseum, 11; 18; pl. XXIII (3); $\mathrm{PM} \mathrm{I}^{2}, 679$.

33 Vgl. zu ähnlichen Phrasen G. Vittmann, Altägyptische Wegmetaphorik, Beiträge zur Ägyptologie 15, Wien 1999, 37ff.

${ }^{34}$ Sicher zu $[s]{ }^{\complement} n h$ zu ergänzen.

${ }^{35}$ Üblich wäre $h r j b . t \underline{n}$ und nicht $t p j b . t n$. 


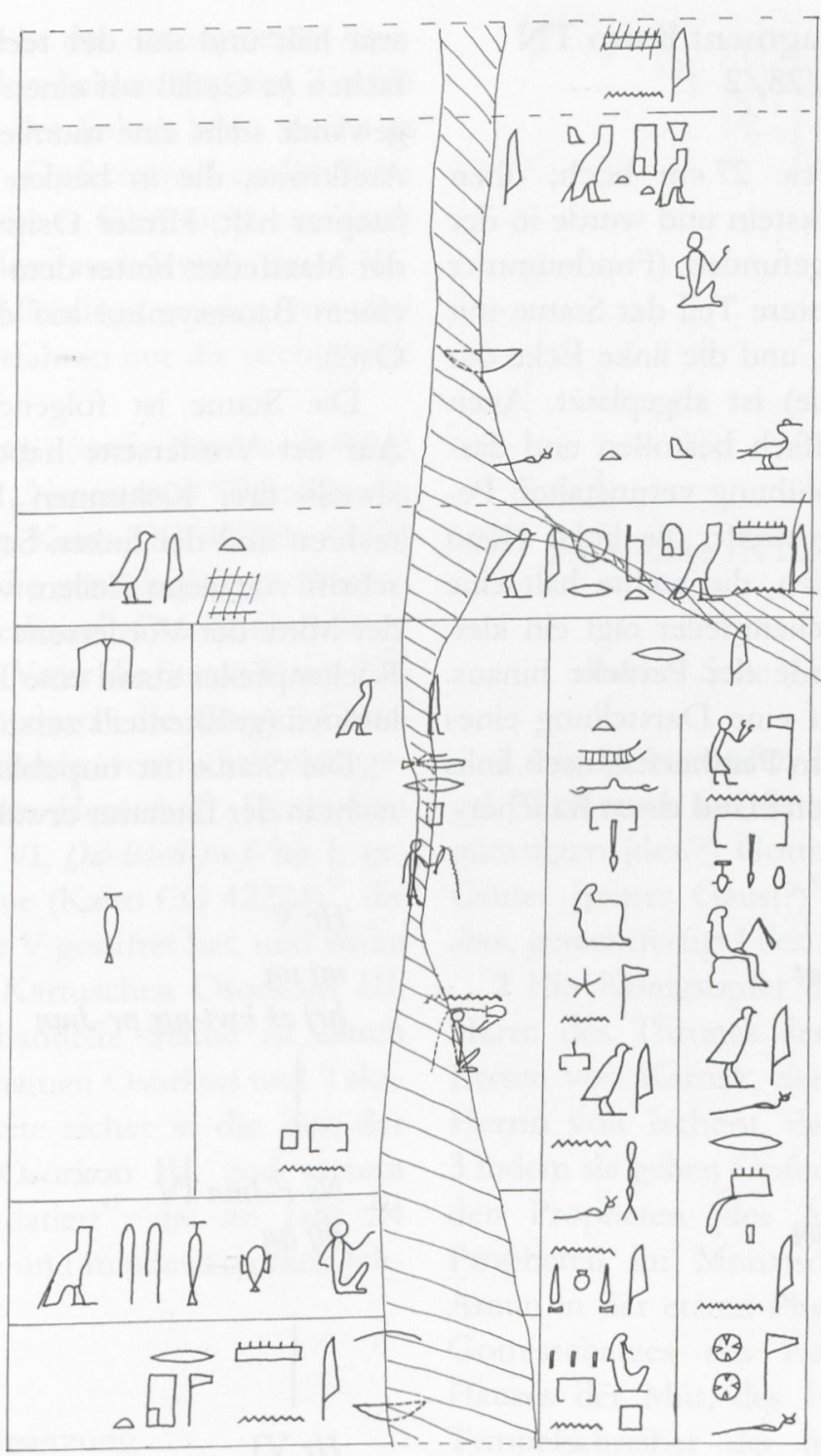

Fig. 7. Kairo TN 20/2/25/1, Rückseite

\section{Rückseite (Fig. 7, Tf. XII):}

Ein großer Teil ist unlesbar, auffällig ist die ungewöhnliche Disposition der Inschrift, für die ich keine Parallele wüsste. Aufgrund des (auseinandergezogenen) Wortes ${ }^{C} q . t(j) . f(j)$ im oberen Drittel könnte es sich in diesem Teil des Textes um einen Anruf an die Lebenden handeln. Im Zusammenhang lesbar ist nur die durch Linien abgesetzte Sicherungsformel rechts in der unteren Hälfte:

„Wer Gott für (mich) preist und ein Totenopfer darbringt 〈für〉 (meine) Statue, wird geehrt werden $^{36}$ durch seinen Stadtgott; wer (aber) kein Totenopfer darbringt 〈für〉 (meine) Statue ${ }^{37}$ und (nicht) Gott für (mich) preist, mit dem werde (ich) zusammen gerichtet werden ${ }^{38}$ durch das (Götter-)Kollegium dieses Hauses.“

${ }^{36} \mathrm{Zu}$ transitivem Gebrauch von $\mathrm{jm} 3 \mathrm{~h}$ in dieser Zeit s. Jansen-Winkeln, Ägyptische Biographien, 17-8.

Die jeweils auf $p r$-hrw folgende Figur kann eigentlich nur eine Schreibung für "Statue“ (znn bzw. hzjj) sein. Es ist natürlich sehr zweifelhaft, davor zweimal ein $<n>$ zu ergänzen, aber ich sehe keinen anderen Ausweg.

${ }^{38}$ Vgl. Edel, Altäg. Gramm., \695; 937. 


\section{Würfelhockerfragment Kairo TN $20 / 2 / 25 / 2$}

Das Fragment (noch ca. $27 \mathrm{~cm}$ hoch; oben $16 \mathrm{~cm}$ breit) ist aus Kalkstein und wurde in der Cachette von Karnak gefunden (Fundnummer K.652). Der gesamte untere Teil der Statue mit Sockel und Füßen fehlt, und die linke Ecke der Vorderseite (linkes Knie) ist abgeplatzt. Auch sonst ist die Statue vielfach bestoßen und dasGesicht durch Salzausblühung verunstaltet. Perücke und Bart sind gestreift, die linke Hand liegt flach auf den Knien, die rechte hält eine Pflanze. Der breite Rückenpfeiler ragt ein kleines Stück über das Ende der Perücke hinaus. Auf der Vorderseite ist eine Darstellung eines kahlköpfigen Priesters im Pantherfell, nach links gewandt, der in der linken Hand einen Räucher-

$H r$ IX

[hm-ntr $n$ Jmn] $m$ Jpt-swt $z \check{s} \breve{s} \iota^{\complement}{ }^{c}-r s j$

$N b-n \underline{t} r w$ IV

[hm-ntr n] Jmn m Jpt-swt $z \check{s} \breve{s} t e^{e}-r s j$

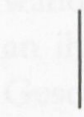

T3-prt

$n b t-p r$

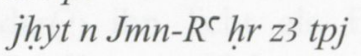

arm hält und mit der rechten mit einem dreifachen $h z-G e f a ̈ ß$ auf einen Altar libiert. Ihm zugewandt steht eine mumienförmige Gestalt mit Atefkrone, die in beiden Händen Wedel und Szepter hält. Hinter Osiris steht eine Frau mit der Maatfeder, hinter dem Priester eine Frau mit einem Baumsymbol auf dem Kopf (West und Ost?).

Die Statue ist folgendermaßen beschriftet: Auf der Vorderseite haben Gott und Priester jeweils drei Kolumnen Beischriften, auf der rechten und der linken Seite ist jeweils eine Inschrift von neun Zeilen, von denen die erste in der Mitte der Vorderseite beginnt, und auf dem Rückenpfeiler stand eine Inschrift von vier Kolumnen (größtenteils zerstört).

Die Statue ist unpubliziert und wird $\mathrm{m}$. W. nicht in der Literatur erwähnt.

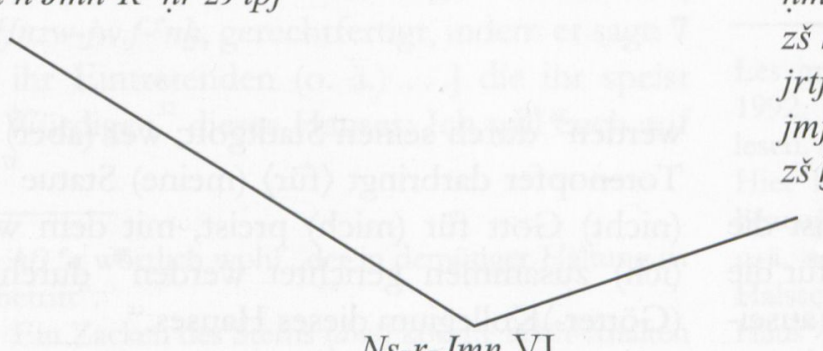

Ns-r-Jmn VI

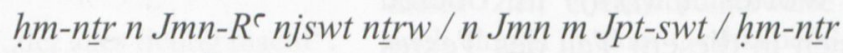

jrtj njswt $m$ Jpt-swt

hmm-ntr jmj $3 b d$.f pr-Jmn hr z 3 tpj

zš htmt-ntr (n pr-Jmn pr-Mwt pr-Hnzw)

jmj-st- ${ }^{-}($?) $p r-J m n$ pr-Mwt pr-Hnzw

zš hwt-nțr $n$ Mwt wrt nb(t) Jك̌rw

Fig. 8. Familie des Neseramun VI 
Besitzer der Statue ist der Amunpriester Ns-r$J m n\left(, \mathrm{VI}^{\circ}\right)$, und in den beiden längeren Texten auf der rechten und der linken Seite macht er nach der einleitenden Opferformel ausführliche genealogische Angaben: er führt nicht nur Vater und Mutter auf, sondern auch jeweils deren Vater und Großvater. Allerdings werden von den Titeln der älteren Vorfahren nur die wichtigsten erwähnt (s. Fig. 8).

Zur Identifizierung (und Nummerierung) dieser Personen der „Neseramun“-Familie, die v. a. von den Statuen Kairo CG 42219-42224 her bekannt sind, s. die genealogischen Studien von Kitchen ${ }^{39}$ und Bierbrier ${ }^{40}$. Der Besitzer Ns-r $J m n$ VI hat für seinen Vater die Statue Kairo CG 42223 gestiftet $^{41}$, die „durch die Gunst“ Osorkons III. aufgestellt wurde, vermutlich eher gegen Beginn von dessen Herrschaft. Denn dem Bruder des Ns-r-Jmn VI, Dd-B3stt-jw. f- $n h$ I, gehört eine weitere Statue (Kairo CG 42224) ${ }^{42}$, die dessen Sohn Ns-r-Jmn V gestiftet hat, und sie ist gleichfalls durch die Kartuschen Osorkons III. datiert. Die hier behandelte Statue ist durch die parallelen Königsnamen Osorkon und Take[loth] auf der Rückseite sicher in die Zeit der Koregenz zwischen Osorkon III. und seinem Sohn Takeloth III. datiert ${ }^{43}$, die im Jahr 24 Osorkons III. begann und mindestens fünf Jahre dauerte.

\section{Übersetzung}

\section{Vorderseite (Fig. 9, Tf. XIII):}

(vor Osiris:) Osiris, der Erste des Westens, der Herr von Abydos,

(vor Priester:) der Opferspeisen gibt dem Propheten Ns-Jmn, gerechtfertigt.

\footnotetext{
${ }^{39}$ Kitchen, Third Intermediate Period, \ 166; 170.

${ }^{40}$ Bierbrier, The Late New Kingdom in Egypt,

${ }^{41}$ Legrain, Statues et statuettes, III, 52-54; pl. 30.

${ }^{42}$ Ibid., 54-57; pl. 31

${ }^{43}$ Auch der Würfelhocker Kairo CG 42211 ist durch die Kartuschen Osorkons III. und Takeloths III. in diese Zeit datiert, s. Jansen-Winkeln, Ägyptische Biographien, 470.
} $68-73$.

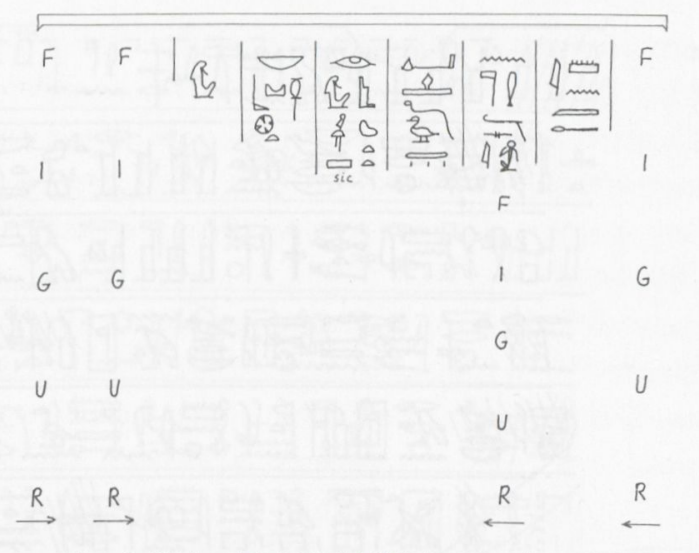

Fig. 9. Kairo TN 20/2/25/2, Vorderseite

Rechte Seite (Fig. 10, Tf. XIV):

1 Ein Königsopfer, Brot, Bier, Rind, Geflügel, Weihrauch auf dem Feuer(?) ${ }^{44}$ für den Begünstigten [des(?) Gottes] seiner Stadt und der Götter [seines Gaus(?) ${ }^{45}$, den Propheten Ns-rJmn, gerechtfertigt,] des Amun in Karnak.

2 Ein Königsopfer des Amonrasonther, des Herrn des Thrones der 〈Beiden〉 Länder, des Ersten von Karnak, der Mut, der Großen, der Herrin von Ischeru, der Herrin der Maat(?) ${ }^{46}$, 3 indem sie geben Opfergaben und Speisen [für] den Propheten [des Amun] in Karnak, den Propheten im Monatsdienst des Hauses des Amun in der ersten Phyle, 4 den Schreiber des Gottesschatzes des Hauses des Amun, des Hauses der Mut, des Hauses des Chons, den Tempelschreiber der Mut, der Großen, der Herrin von Ischeru, den Großen seines Tragschreins, (nämlich) des Königs, des Gottes und Herrschers von Heliopolis 5 Ramses (III.) ${ }^{47}$

${ }^{44}$ Vgl. Z. 1 und 3 der Inschrift der linken Seite.

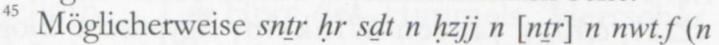
hzjj) $n$ ntr $w$ [sp3t.f hm-ntr $\left.N s-r-J m n ~ m 3^{c}-h r w\right]$ zu verstehen bzw. zu ergänzen (vgl. auch linke Seite, Z. 1). In diesem Fall wäre $h z j j$ der Gottesbezeichnung $n t r r ~ n n w t . f$ nachgestellt. Ebenso möglich wäre natürlich ,für den Gott seiner Stadt und den Begünstigten der Götter [seines Gaus o. ä., den Propheten ... .]".

${ }^{46} \mathrm{Zu} n b(t) \quad m 3{ }^{c} t$ als Beiwort der Mut s. Ch. Leitz (ed.), Lexikon der ägyptischen Götter und Götterbezeichnungen, IV, OLA 113, 2002, 60-1.

${ }^{47}$ Möglicherweise ist das Suffix $f$ nach $q n j$ auch zu streichen (die Lesung ist sicher). Auf der linken Seite, Z. 4-5 lautet der Titel $3 n$ qnj njswt $R^{\ulcorner}-m s j-s w h q 3$ Jwnw. 


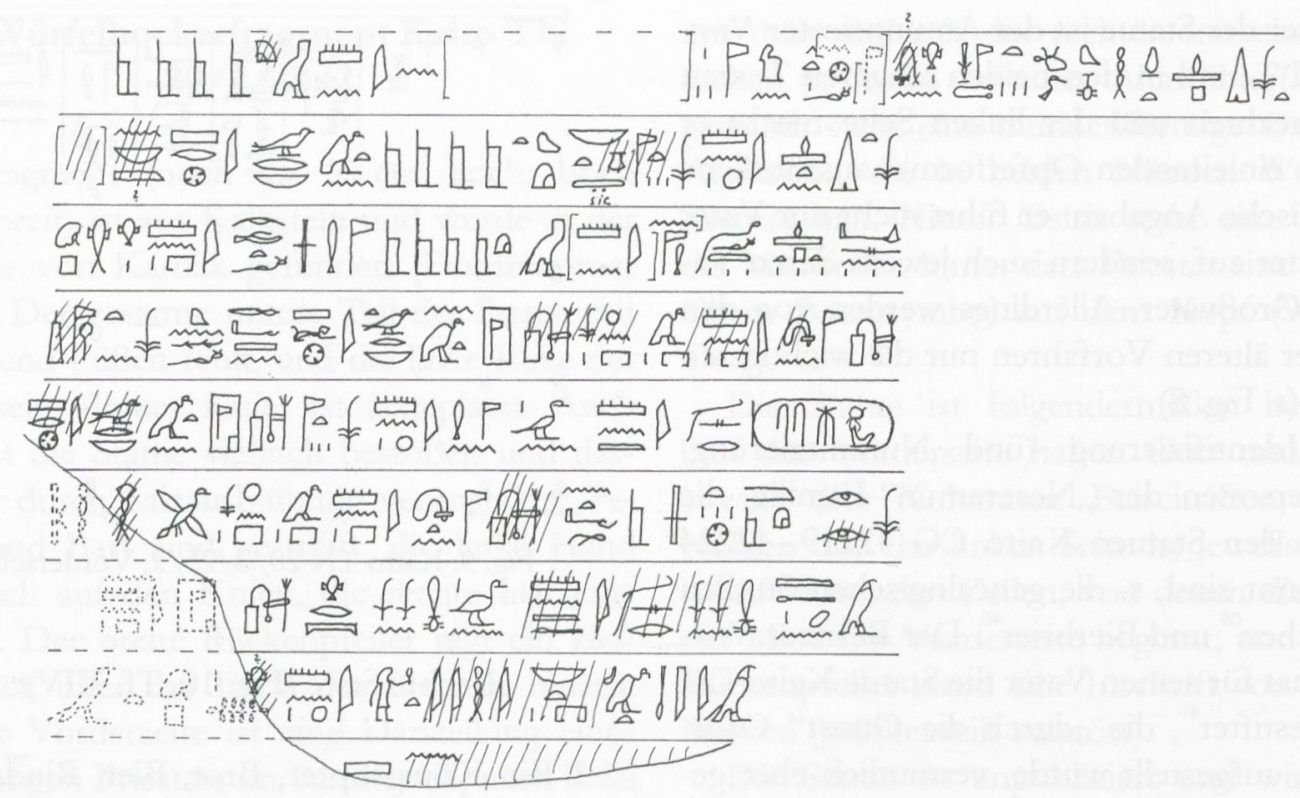

Fig. 10. Kairo TN 20/2/25/2, rechte Seite

Ns-r-Jmn (VI), gerecht $\langle$ fertigt $\rangle$, den Sohn des Propheten des Amonrasonther, des Tempelschreibers der Mut, der Großen, der Herrin von Ischeru, 6 der ,Augen des Königs' in Karnak, des diensttuenden Priesters, des Schreibers des Gottesschatzes des Hauses des Amun, des Hauses der Mut, des Hauses des Chons von der Station $^{48} 7 \mathrm{Hr}$ (VI), gerechtfertigt, des Sohnes des gleichrangigen Ns-r-Jmn (IV), des Sohnes des Obersten der [Tempel]schreiber [des Hauses des Amun] $8 \mathrm{Hr}(\mathrm{V})$, gerechtfertigt, seine Mutter

Der Vater $H r$ VI führte ihn auch: Auf dem Würfelhocker Kairo CG 42222, f,2 (Legrain, Statues et statuettes, III, 52) heißt er 3 n qnj $n$ njswt Hq3-Jwnw [in Kartusche], auf CG 42224, n,5-6 (ibid., 56) 3 qnj $n$ njswt $R^{e}-m s j-s w h q 3-J w n w$ [in Kartusche]. Auch der Urururgroßvater des $N s-r-J m n$ VI namens $H r$ III ist 3 qnj $n$ Hq3-Jwnw [in Kartusche] (Würfelhocker Kairo CG 42221, c,10, s. Jansen-Winkeln, Ägyptische Biographien, 537); bei ihm folgen dort noch ähnliche Funktionstitel: [hm-ntr $n$ p3 s] hrj ntrw $n$ P3j-ndm $m 3^{\top}-h r w$ und $w^{c} b$ n $p 3$ jbjb $n$ Wsrkn (in Kartusche). Auf einem Sargfragment aus Medinet Habu ist nur noch die abschlieBende Kartusche (Hq3-Jwnw) dieses Titel erhalten; es könnte zu $H r$ III oder VI gehören, s. Anthes in: Hölscher, Medinet Habu, IV, 25. Zur Bedeutung des Titels s. J. Leclant, Enquêtes sur les Sacerdoces et les sanctuaires égyptiens, BdE 17, 1954, 21-3 (m); Helck, Materialien, 119-22 (a).

${ }^{48}$ Zum Zusatz p3 w3h vgl. zuletzt Jansen-Winkeln, CdE 78, 2003, 41-2(11). ist die Hausherrin und Musikerin des Amun-Re in [der ersten Phyle T3-prt] 9 [...].

Linke Seite (Fig. 11, Tf. XV):

1 Ein Königsopfer, Brot, B〈ier〉, Rind, Geflügel, Weihrauch [auf dem Feuer(?) für (den Gott $\mathrm{NN})$ ], indem er gibt ein Totenopfer und Weihrauch auf dem Feuer für den Propheten Ns-r$J m n$, gerechtfertigt, des Amun in Karnak ${ }^{49}$.

2 Ein Königsopfer des Amun-Re, des Urzeitlichen der Beiden Länder, mit heiligem Arm, der das Seiende gemacht und alles, was ist, geschaffen hat, (und) des Chons in Theben(?), Neferhotep, 3 indem sie geben ein Totenopfer, Weihrauch auf dem Feuer, die angenehme Luft des Nordwinds an die Nase des Propheten des Amonrasonther, der "Augen des Königs" in Karnak, 4 des Schreibers des Gottesschatzes, des diensttuenden Priesters des Hauses des Amun, des Hauses der Mut und des Chons, des Tempelschreibers der Mut, der Großen, der Herrin von Ischeru, des Großen des Tragschreins 5 des Königs Ramses (III.), des Gottes, des Herrschers von Heliopolis, Ns-r-Jmn (VI),

${ }^{49}$ Formulierung und Struktur der kurzen Opferformeln in Z. 1 der linken wie der rechten Seite sind sehr ungewöhnlich. 


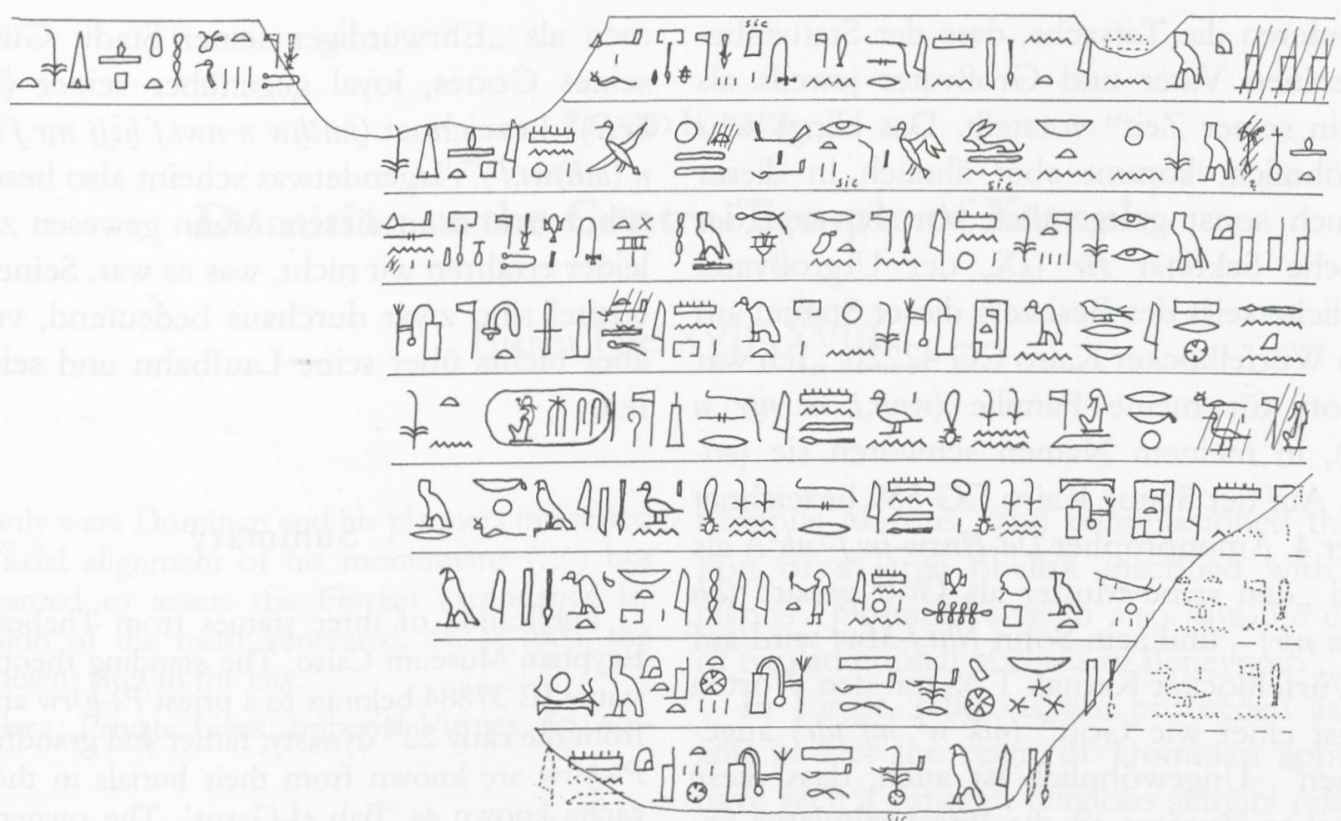

Fig. 11. Kairo TN 20/2/25/2, linke Seite

gerechtfertigt, des Sohnes eines gleichrangigen, eines Gottes in seiner Zeit, Sohn eines Gottes 6 in seiner Zeit ${ }^{50}, H r j(\mathrm{VI})$, gerechtfertigt, des Sohnes des gleichrangigen Ns-r-Jmn (IV), gerechtfertigt, des Sohnes eines gleichrangigen, des Obersten der Tempelschreiber des Hauses des Amun $7 \mathrm{Hrj}(\mathrm{V})$, gerechtfertigt; seine Mutter ist die Hausherrin und Musikerin des Amun-Re in der ersten Phyle T3-prt, [die Tochter des Propheten] des $\mathbf{8}$ Amun $^{51}$ in Karnak, des Sekretärs des Südens $N b$-ntrw (IV), gerechtfertigt, [des Sohnes des Propheten des 9 Amun] in Karnak, des Sekretärs des Südens $H r j$ (IX), [gerechtfertigt].

Rückseite (Fig. 12, Tf. XVI):

rechts:

1 König von Ober- und Unterägypten Osork $[\mathrm{on}]^{52} \ldots$ Horus, mit kräf[tigem $\mathrm{Arm}^{53} \ldots$ ]

${ }^{50}$ Die Stelle lässt sich wohl nicht anders verstehen, als dass der Verfasser hier seinen Vater und Großvater metaphorisch als „Gott in seiner Zeit", also für seine Umgebung beschreibt, s. dazu unten.

Zur Lesung Barke mit Sonnenscheibe als Jmn s. Drioton, RdE 12, 1960, 89-90.

${ }^{52}$ Außer der Kolumne ganz rechts ist die gesamte Inschrift der Rückseite schwer beschädigt und kaum zu entziffern. Es ist aber genug erhalten, um die Identifi-

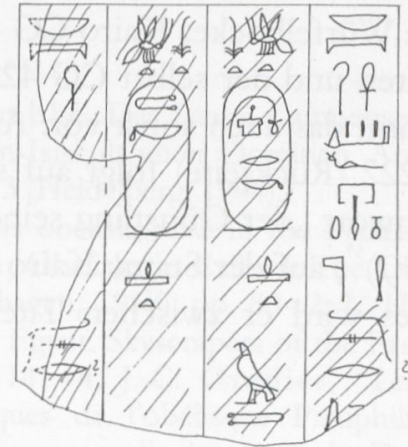

Fig. 12. Kairo TN 20/2/25/2, Rückseite

2 der Herr des Himmels, der Herrscher der Lebenden, indem er gibt ein Totenopfer $\langle$ für $\rangle$ den Propheten Ns-r-J[mn ... . .

\section{links:}

3 König von Ober- und Unterägypten Take[loth ... ] 4 Herr des Himmels, Herrscher [der Lebenden, indem er gibt ein Totenopfer $\langle$ für $\rangle$ den Propheten] Ns-r-[Jmn . . .].

Bemerkenswert an dieser Statue ist zum einen, dass sie einen weiteren Beleg für die Koregenz Osorkons III. und Takeloths III. liefert,

zierung der beiden Königsnamen als Osorkon und Takeloth zu sichern.

${ }^{53} \mathrm{Ob} \mathrm{Hr} \underline{t}[\mathrm{~m} 3-\mathrm{]}$ zu ergänzen? 
zum anderen die Tatsache, dass der Statuenbesitzer seinen Vater und Großvater jeweils als „Gott in seiner Zeit" vorstellt. Das klingt sehr ungewöhnlich, kommt aber ähnlich in dieser Zeit auch sonst gelegentlich vor. So sagt der königliche Sekretär $\mathrm{Hr}$ (IX, der Urgroßvater mütterlicherseits des Besitzers dieser Statue) auf seinem Würfelhocker Kairo CG 42226: „Ich war ein Gott für meine Familie (wnn.j $m$ ntr $n$ $m h w t . j$ ), in meinem Namen schworen sie (sogar) ${ }^{6.54}$. Auf der Statue Kairo CG 559 bezeichnet sich der 4. Amunprophet $D d-H n z w-j w . f-n h \mathrm{~A}$ als jemand „den seine Mutter als Gott gebar" (bhh tm3t.f $m n t r)^{55}$, und sein Sohn Nht.f-Mwt wird auf dem Würfelhocker Karnak T 35 mit den Worten „du bist einer wie Gott" (ntk w $w^{r}$ mj ntr) angesprochen $^{56}$. Ungewöhnlich ist auch, dass diese eulogischen Zusätze an die Priestertitulatur angehängt sind, dort erwartet man keine „biographischen" Beiworte. Aber bei eben diesem $\mathrm{Hr}$ VI, dem die Würfelhocker Kairo CG 42222 und 42223 gehören und der selbst CG 42219 gestiftet hat, kommt das auch sonst vor: Auf der Statue CG 42222 (Rückseite) folgt auf seinen Namen das Beiwort „der Günstling seines Gottes“ (p3 hzjj $n n t r . f)^{57}$, auf der Statue Kairo CG 42224 eines Sohnes wird er zwischen Titel und $\mathrm{Na}$ - men als „Ehrwürdiger seiner Stadt, Günstling seines Gottes, loyal gegenüber seiner $\langle\mathrm{Fa}\rangle\rangle_{\mathrm{mi}}-$ lie(?) “ bezeichnet (jm3hww n nwt.f hzjj ntr.f mnh-jb $n\langle m h\rangle w t . f)^{58}$. Irgendetwas scheint also besonders rühmenswert an diesem Mann gewesen zu sein, leider erfahren wir nicht, was es war. Seine Priestertitel sind zwar durchaus bedeutend, verraten aber nichts über seine Laufbahn und seine Taten.

\section{Summary}

Publication of three statues from Thebes in the Egyptian Museum Cairo. The standing theophorous statue JE 37884 belongs to a priest $P 3-h 3 r w$ and dates from the early $22^{\text {nd }}$ dynasty; father and grandfather of P3-h3rw are known from their burials in the coffin cache known as 'Bab el-Gasus'. The owner of the block statue TN $20 / 2 / 25 / 1$ is the fourth prophet of Amun Dd-Hnzw-jw.f- $n h$ C, a son-in-law of king Takeloth II. and a grandson of both king Harsiese A and the famous Theban priest Nakhtefmut A. He is well-known from another block statue (Cairo CG 42211) dated by the cartouches of Osorkon III. and Takeloth III. These same cartouches are found on the third statue, the block statue fragment TN $20 / 2 / 25 / 2$ of Ns-r-Jmn VI, a member of the prominent Theban Neseramun family. None of the three statues has been published before.

\footnotetext{
$511(\mathrm{k})$.

${ }^{54}$ Jansen-Winkeln, Ägyptische Biographien, 141;

Ibid., 11; 435 (b,5).

Karnak-Nord IV, Fig. 143, Z. 3.

${ }^{57}$ Legrain, Statues et statuettes, III, 52, f,3.
}

\footnotetext{
${ }^{58}$ Ibid., 56, n,6-7; Jansen-Winkeln, Ägyptische Biographien, 299.
} 


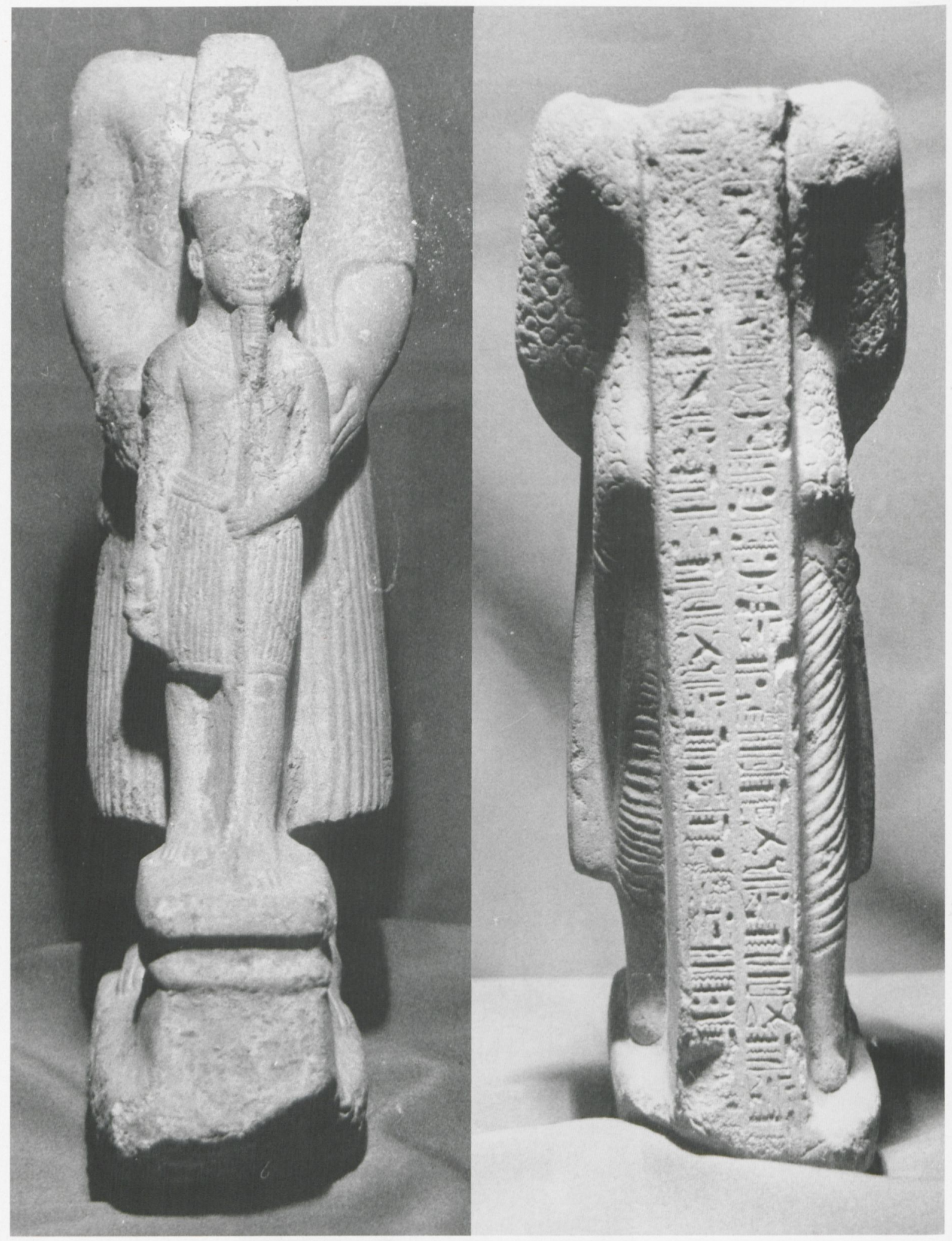

Kairo JE 37884, Vorder- und Rückseite. 


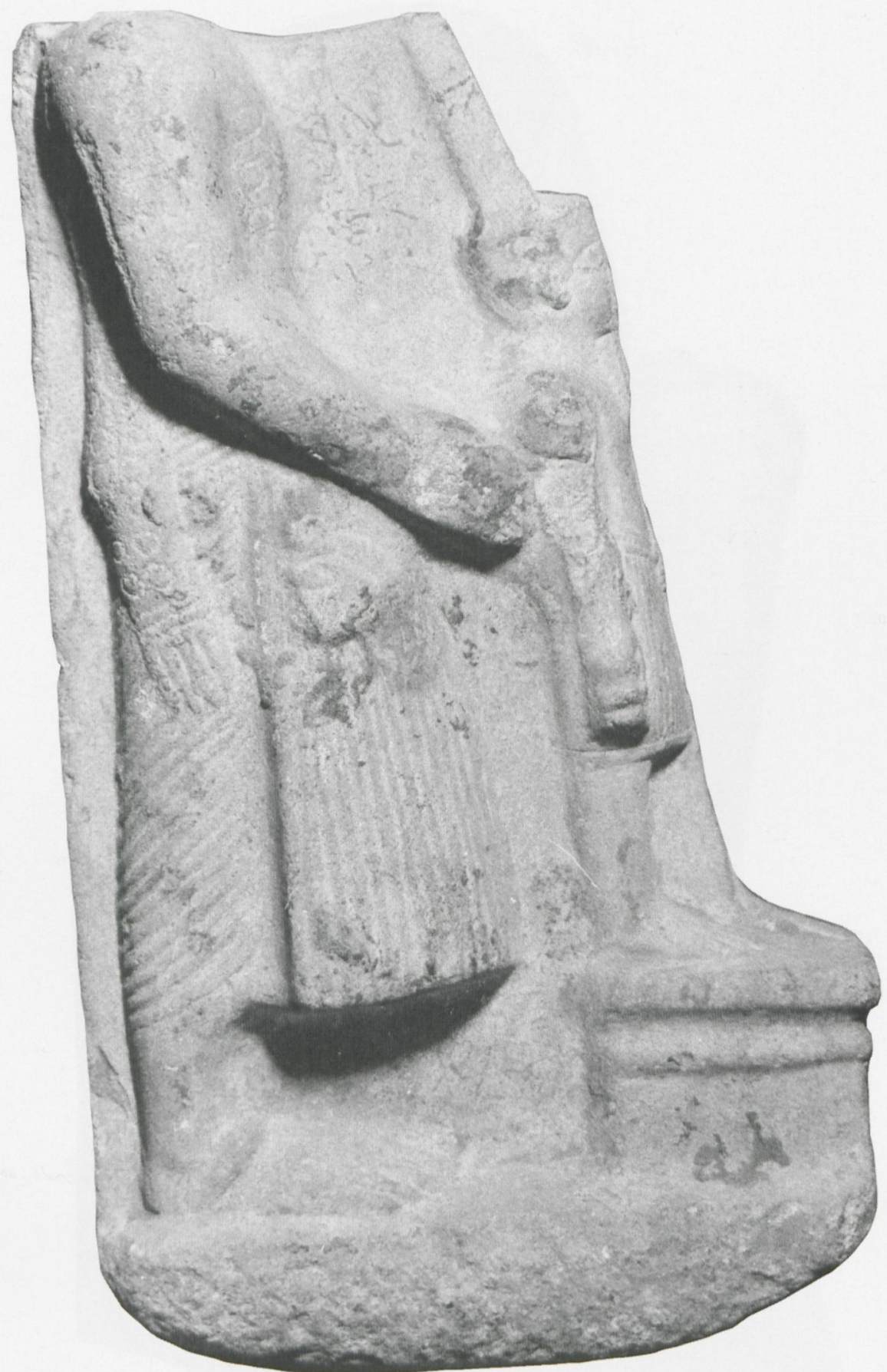

Kairo JE 37884, rechte Seite (zu Jansen-Winkeln, Drei Statuen). 


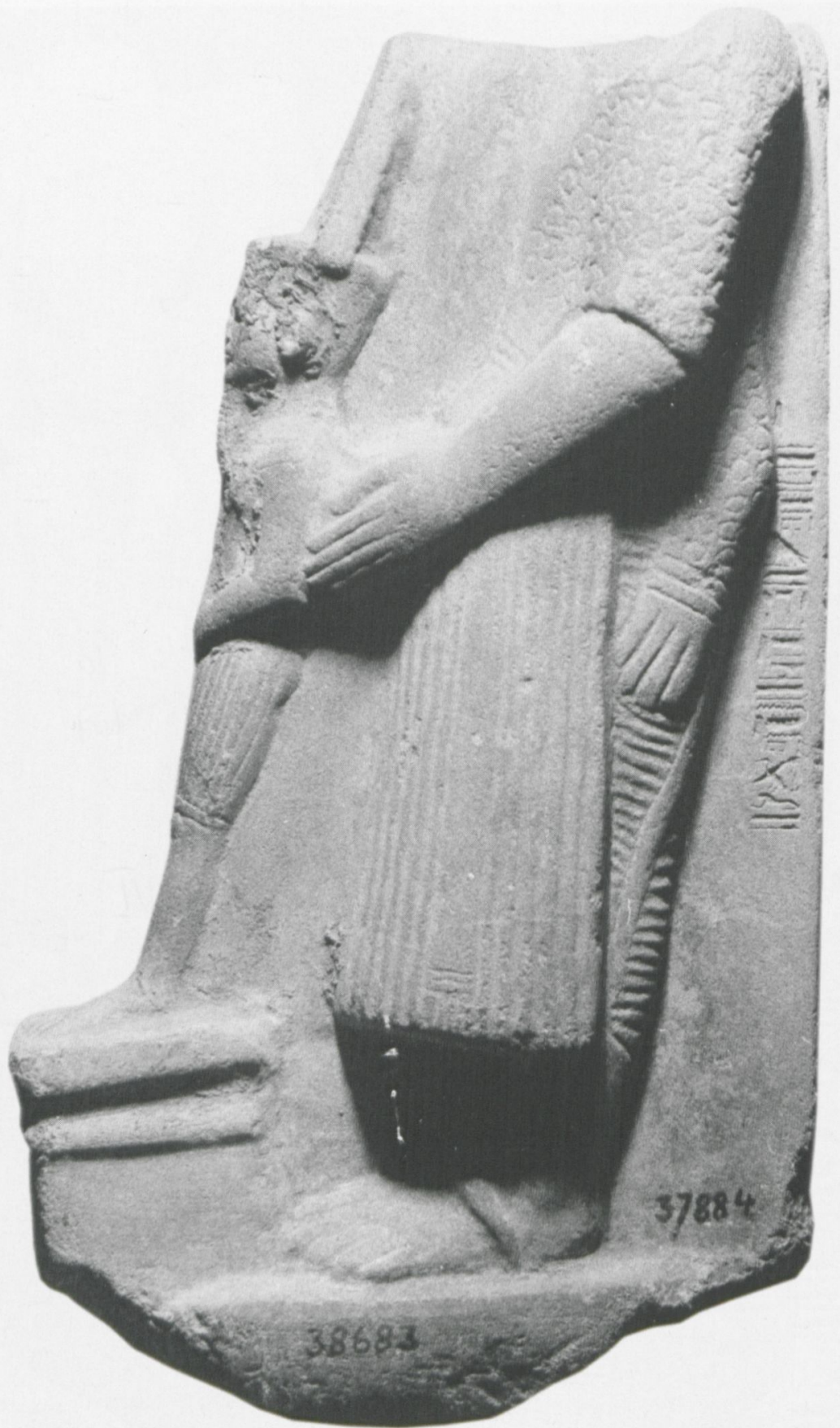

Kairo JE 37884, linke Seite (zu Jansen-Winkeln, Drei Statuen). 
TAFEL IX

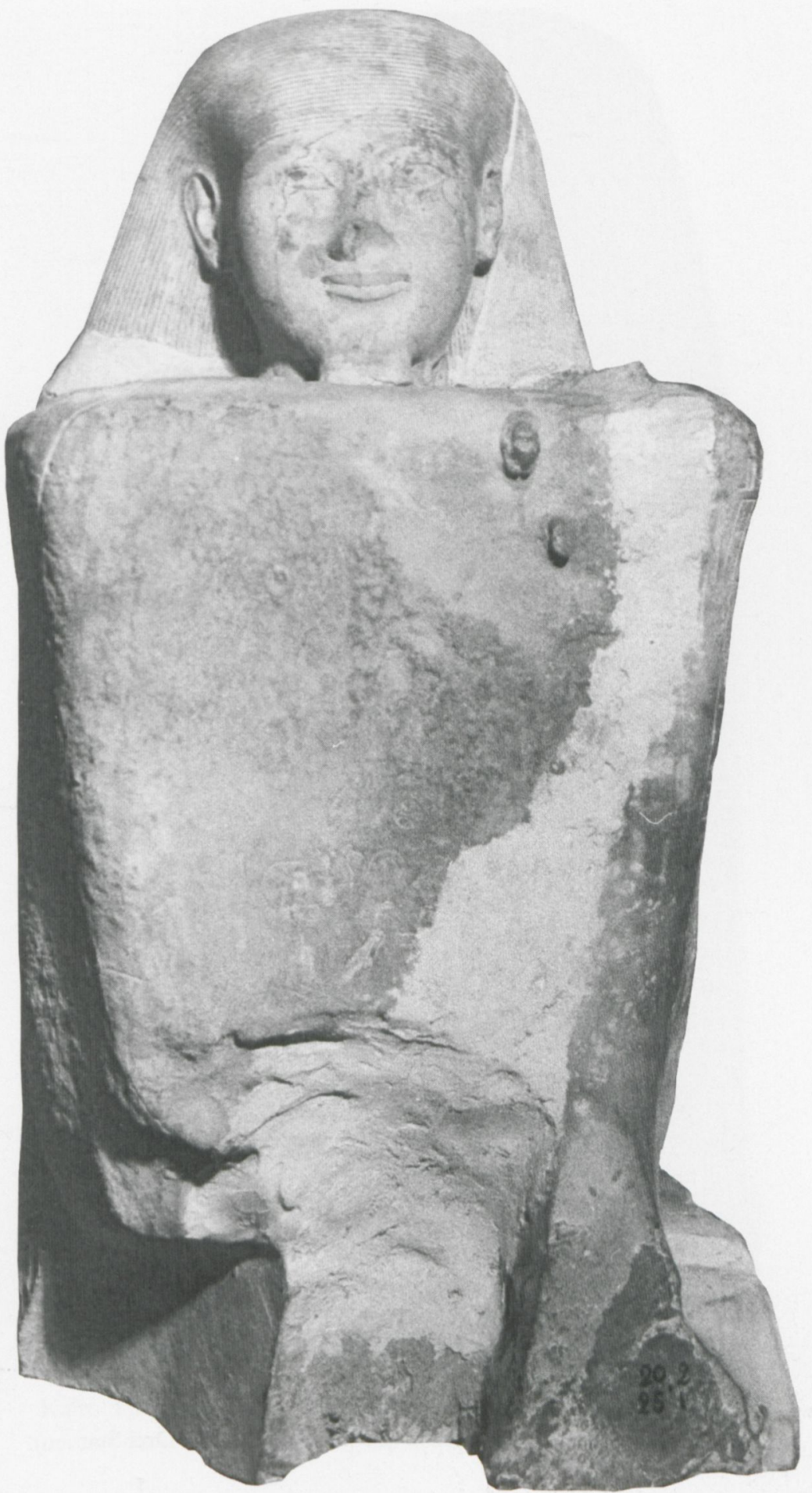

Kairo TN 20/2/25/1, Vorderseite (zu Jansen-Winkeln, Drei Statuen). 
TAFEL $\mathrm{X}$

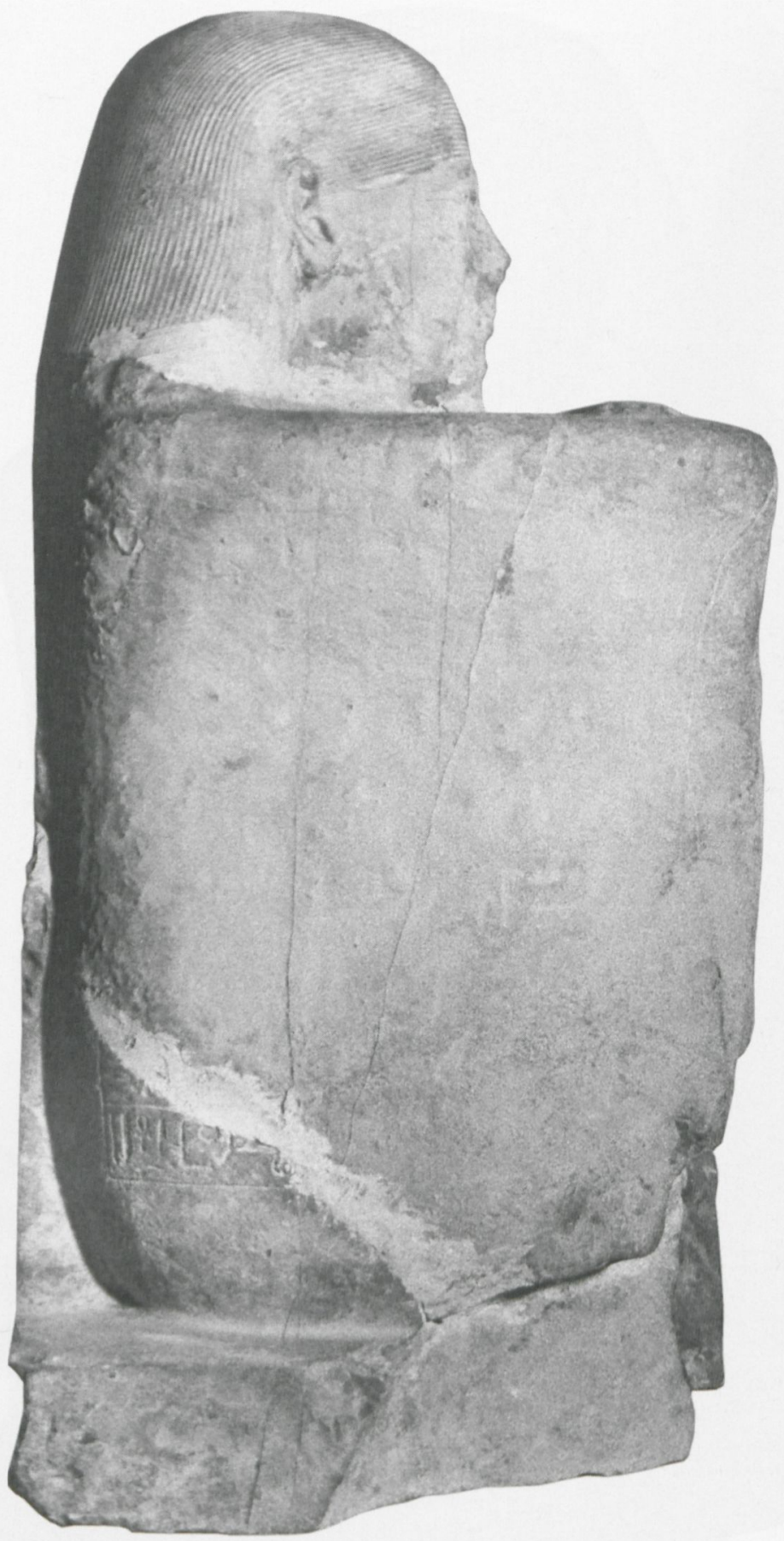

Kairo TN 20/2/25/1, rechte Seite (zu Jansen-Winkeln, Drei Statuen). 
TAFEL XI

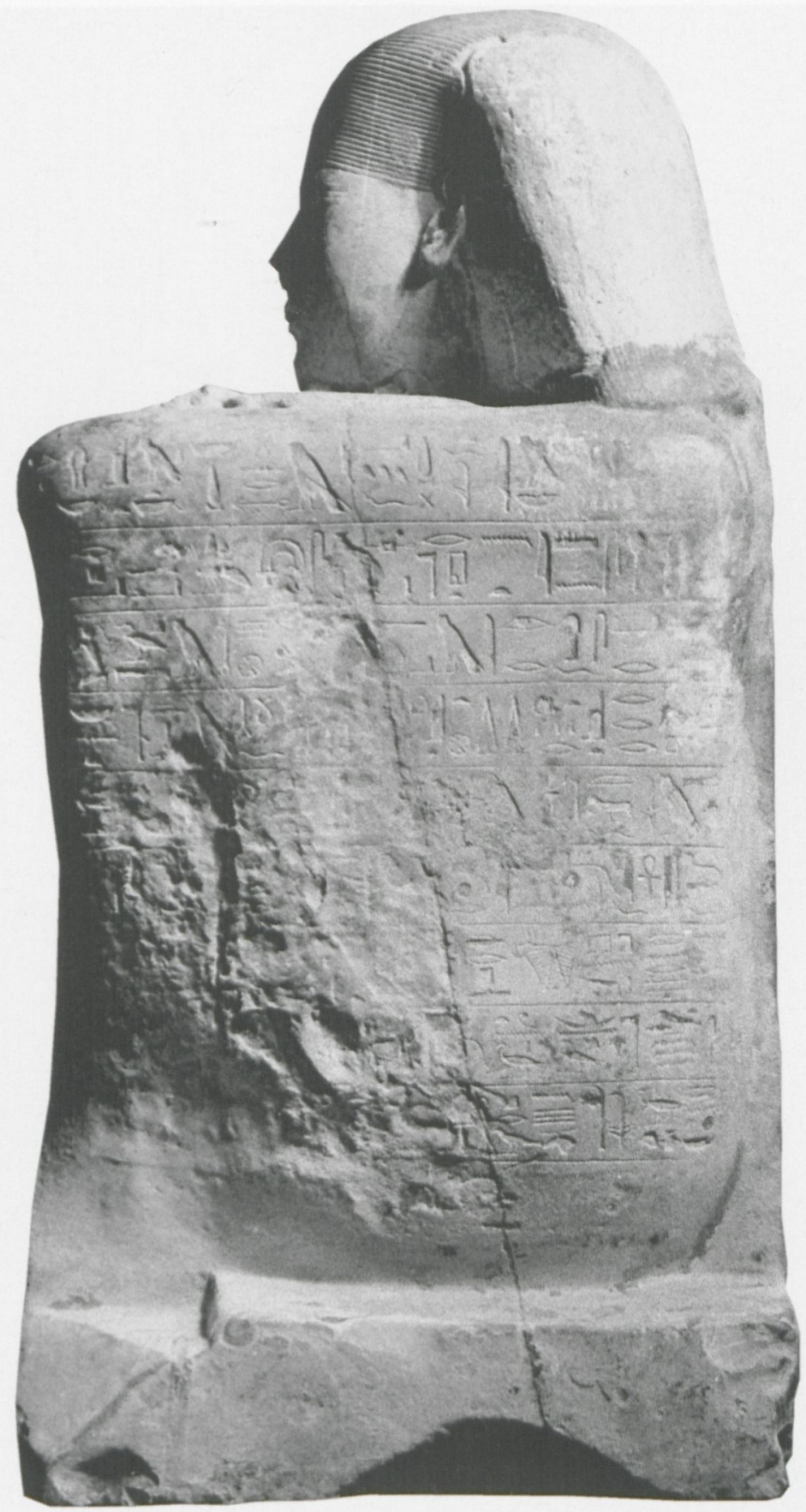

Kairo TN 20/2/25/1, linke Seite (zu Jansen-Winkeln, Drei Statuen). 
TAFEL XII

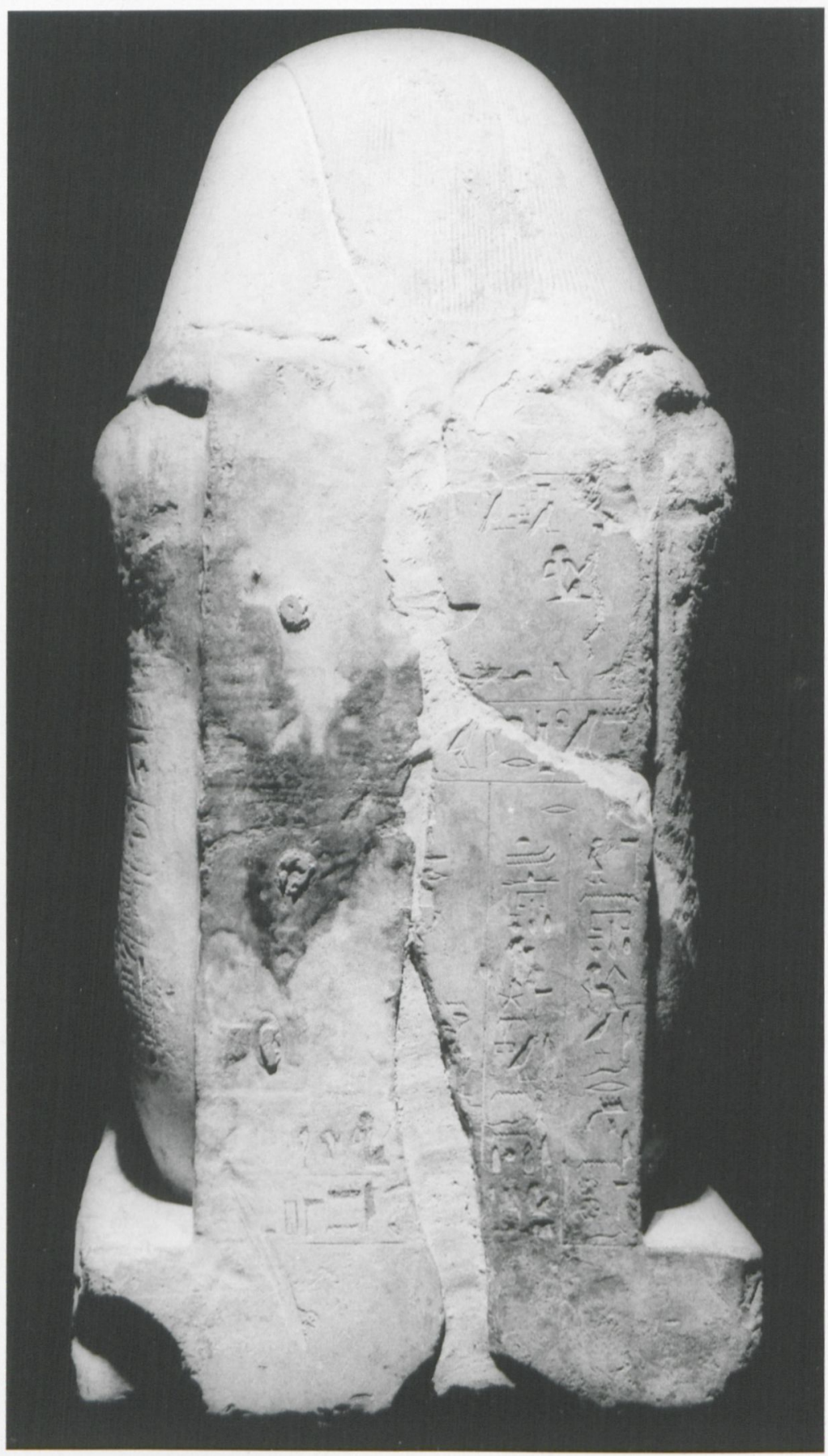

Kairo TN 20/2/25/1, Rückseite (zu Jansen-Winkeln, Drei Statuen). 
TAFEL XIII

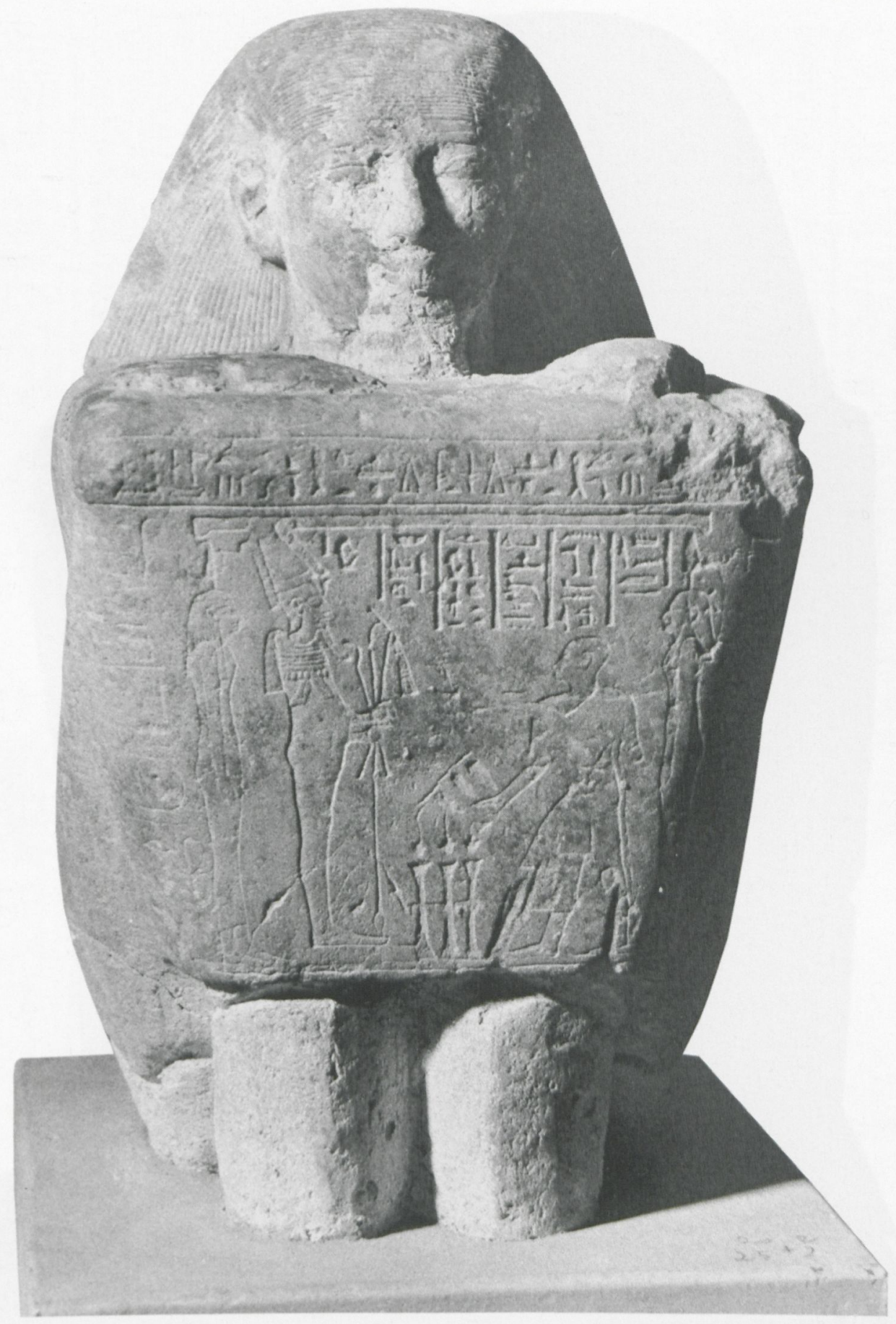

Kairo TN 20/2/25/2, Vorderseite (zu Jansen-Winkeln, Drei Statuen). 


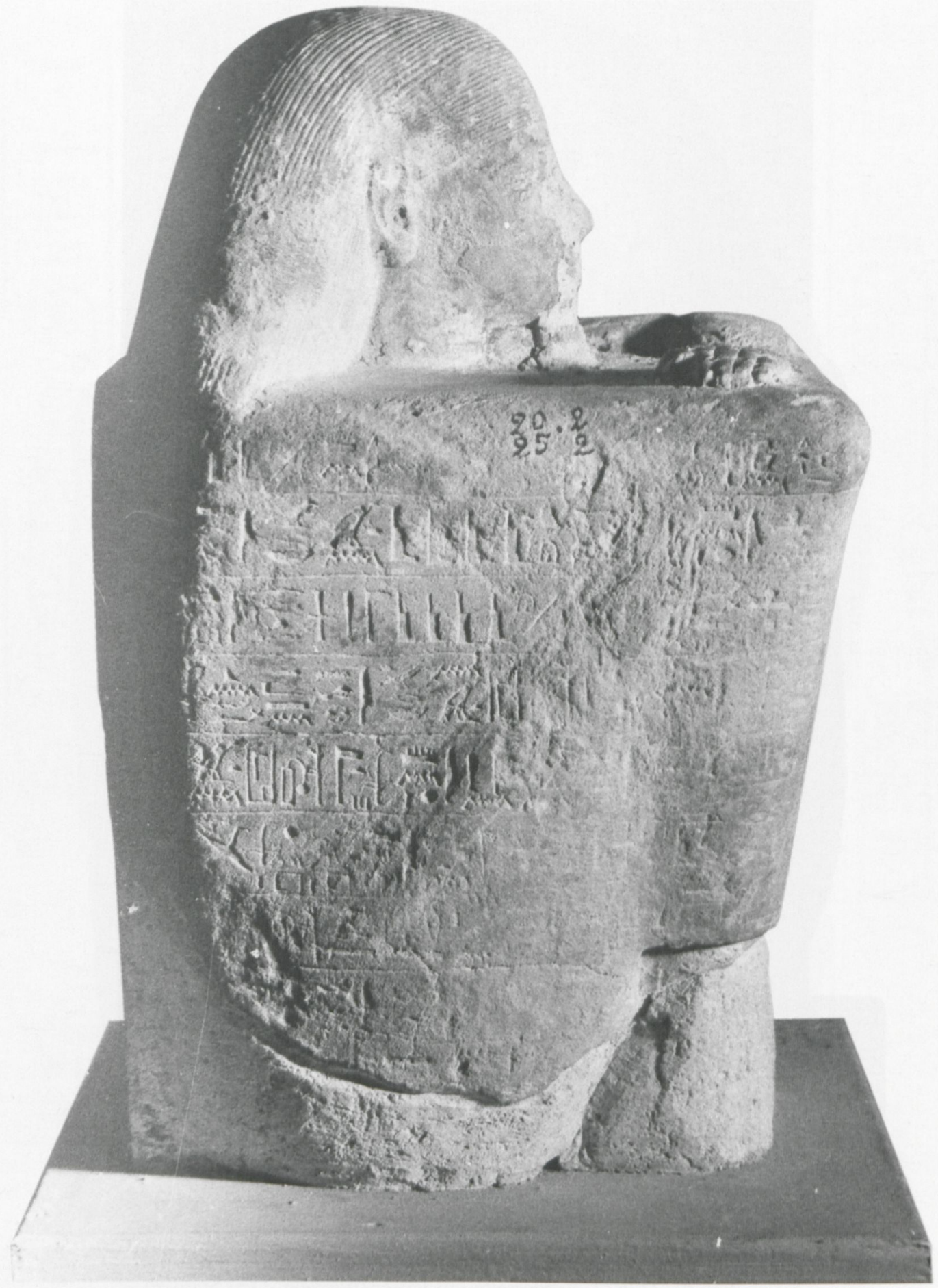

Kairo TN 20/2/25/2, rechte Seite (zu Jansen-Winkeln, Drei Statuen). 
TAFEL XV

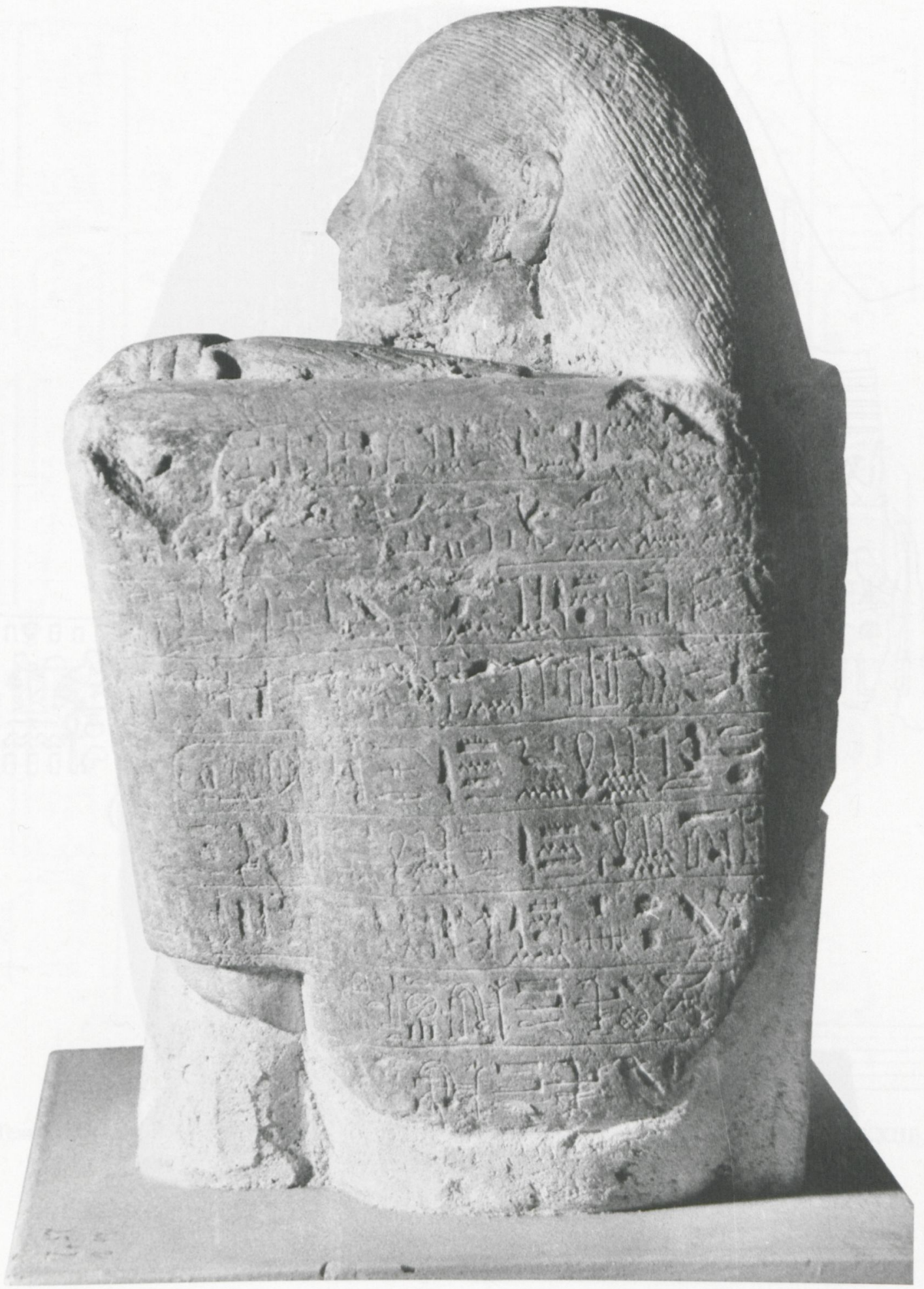

Kairo TN 20/2/25/2, linke Seite (zu Jansen-Winkeln, Drei Statuen). 


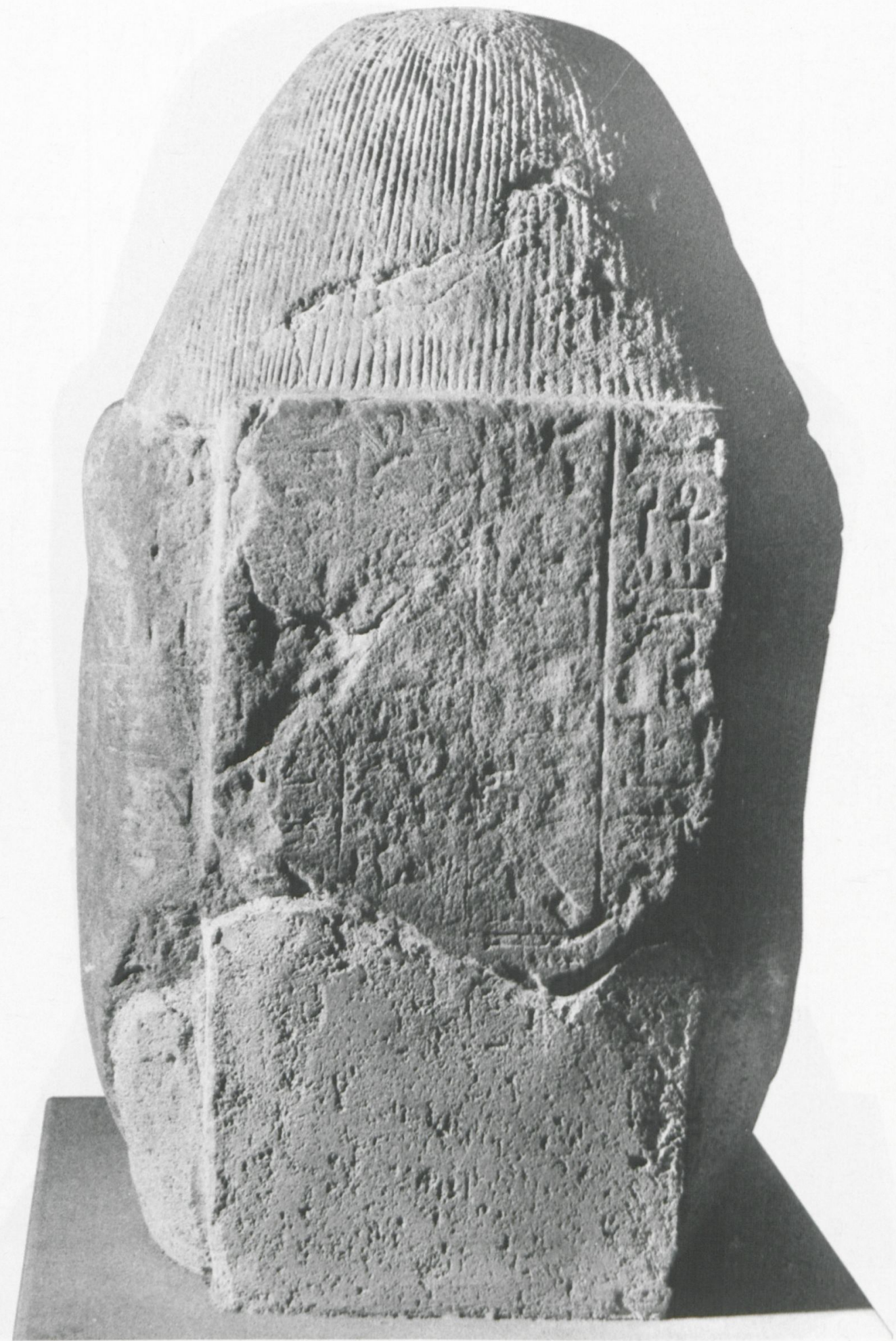

Kairo TN 20/2/25/2, Rückseite (zu Jansen-Winkeln, Drei Statuen). 